Portland State University

PDXScholar

\title{
The Improvement of Machine Learning Accuracies Through Transfer Learning
}

Jordan T. Le

Portland State University

Follow this and additional works at: https://pdxscholar.library.pdx.edu/honorstheses

\section{Let us know how access to this document benefits you.}

\section{Recommended Citation}

Le, Jordan T., "The Improvement of Machine Learning Accuracies Through Transfer Learning" (2019). University Honors Theses. Paper 699.

https://doi.org/10.15760/honors.716

This Thesis is brought to you for free and open access. It has been accepted for inclusion in University Honors Theses by an authorized administrator of PDXScholar. Please contact us if we can make this document more accessible: pdxscholar@pdx.edu. 
The Improvement of Machine Learning Accuracies through Transfer Learning

\author{
By \\ Jordan Le
}

An undergraduate honors thesis submitted in partial fulfillment of the

requirements for the degree of
Bachelor of Science
In
University Honors
And
Computer Science

Thesis Adviser

Melanie Mitchell

Portland State University

2019 


\begin{abstract}
Pretrained models could be reused in a way that allows for improvement in training accuracy. Training a model from scratch takes time. The goal is improving accuracy and minimizing the loss across individual epochs. The hypothesis is that transfer learning could potentially improve on the rate of accuracy and speed of training per epoch iteration.
\end{abstract}




\section{ACKNOWLEDGEMENT}

I'd like to take a moment to express my sincerest gratitude to Professor Melanie Michell for her guidance and expertise throughout this process. She proposed the idea of using the weights from the Vgg16 model for research on transfer learning. Not only was she my advisor, but she was also my professor for machine learning. During this process, I have learned so much. Without Professor Michell, this thesis could not have been conducted. So once more, I am exceptionally grateful for all that she's done and everything she's taught me along the way.

I'd also like to acknowledge how this research is build on the foundation of the Kratos project. Our machine learning capstone project proposed by Nike. My personal thanks go out to each of the Capstone team members. 


\section{List of Figures}

1. Figure 1: In this example, this is a CNN that recognizes $2 \mathrm{D}$ arrays of pixelated images as X's or O's. [18]

2. Figure 2: There are a multitude of cases that are considered as X's or O's. The CNN needs to be able to adapt to these images and identify them accordingly. As shown in the slide, the categories have to deal with translation, scaling, rotation, and/or weight. All of these are still classified as X's or O's, but to a computer may be seen as different from the initial pattern. [18]

3. Figure 3: To manage identifying the images that have been slightly altered, the CNN goes about identifying subtle qualities of the classification. In the image to the left, notice the colored boxes in each of the $2 \mathrm{D} X$ arrays. These boxes are the $\mathrm{CNN}$ viewing the image in sub-images and identifying filters. [18]

4. Figure 4: Here, the CNN has identified three filters that are associated with the $\mathrm{X}$ classification. Each of these sub-images are of the dimension 3x3 pixels. Starting from the left, the $\mathrm{CNN}$ has discovered a diagonal line from the top left to the bottom right. In the middle, there is an $\mathrm{X}$ or crossing pattern. Lastly, it found a diagonal, from top right corner to bottom left corner. [18]

5. Figure 5: Now, we see how the CNN looks at the big picture and works its way through to identify where each of the filters are in the image. Starting from the top left corner, the CNN identifies the first diagonal of the $\mathrm{X}$, then the right as it continues to view $3 \times 3$ sub-images along its way through the full 2D array. Eventually the CNN identifies the crossing pattern in the image and continues along its way as it finds the bottom two legs of the X. Notice how each filter is matched to certain sections throughout the image. [18]

6. Figure 6: Here, the CNN finds a matching filter and pattern in the image. As shown by the red and yellow boxes, the red is the corresponding filter pixel. The yellow is the corresponding image pixel. Both of those values are multiplied together to get the window in the top right corner. [18]

7. Figure 7: The next step is taking the $3 \times 3$ window in the top center and taking its mean as shown in the top right corner. This results in a 1 meaning that the pattern is a $100 \%$ match at that position. [18] 
8. Figure 8: The convolution process is applied to each of the filters on the same image. Their resulting matrices are displayed to the right side of the image. As we can see from the image, the filters are projected into their outputs with the diagonal lines, then the cross section. [18]

9. Figure 9: The CNN takes a window of $2 \times 2$ and slides it across the input matrix. Notice how the output is being populated with the maximums of each $2 \times 2$ slide window. This is to preserve the most important aspect of the initial image. [18]

10. Figure 10: Once the max pooling is complete, its output is approximate a quarter the size of the initial input. Note how the key features of the image are still displayed, the diagonal line declining towards the right. [18]

11. Figure 11: Here, we see the result of each filter after we apply the max pooling layer. For the most part, the patterns are more generalized in there simpler forms. This will help keep the computer generalize and be less literal in its analysis of images. [18]

12. Figure 12: Runs through each pixel and if it finds a negative value, it replaces the value with zero. Once complete, notice how the output image is the same size as the initial image. [18]

13. Figure 13: The ReLU is applied to each of the filters to prevent values from approaching infinity and therefore allowing easier computation. [18]

14. Figure 14: The CNN converts the output from the previous layers into a one dimensional list of values. Every value is represented. [18]

15. Figure 15: The 1D list of 12 values then is polled to determine if the image the CNN was passed is an $\mathrm{X}$ or an $\mathrm{O}$. [18]

16. Figure 16: The outputs of one layer are continually passed to the next layer as input. [18]

17. Figure 17: Stacking of layers can be continually added. The general pattern iterates between CONV, ReLU, and POOL before it comes to the Fully Connected or Dense layer in order to classify the input. [18]

18. Figure 18: "Three ways in which transfer might improve learning."[10]

19. Figure 19: All fifty categories of clothing from the Deep Fashion Category Dataset. [8] 
20. Figure 20: Representative images from the Deep Fashion Category Dataset[8]. These images are all varying examples from the Blouse category (Category_label 3).

21. Figure 21: Vgg16's Model Architecture, developed by Oxford. [20]

22. Figure 22: Scratch Model Architecture

23. Figure 23: Transfer Model Architecture

24. Figure 24: Model Architecture Key

25. Figure 25: Top 5 Scratch Model Predictions

26. Figure 26: Top 5 Vgg16 Transfer Model Predictions 


\section{List of Plots and Tables}

1. Plot 1: Loss on Training Set

2. Table 1: Highlights of Loss on training set data.

3. Plot 2: Top 1 Accuracy on Test Set

4. Table 2: Highlights of top 1 accuracy on test data.

5. Plot 3: Top 5 Accuracy on Test Set

6. Table 3: Highlights of top 5 accuracy on test data. 


\section{Table of Contents}

1. INTRODUCTION

2. BACKGROUND

2.1 Input and Output 12

2.2 Training and Loss 12

2.3 Evaluation $\quad 14$

2.4 Neural Networks $\quad 15$

2.5 Convolutional Neural Networks $\quad 15$

2.5.1 Filters and Convolutional Layers 18

2.5.2 Pooling Layer 19

2.5.3 Normalization with Rectified Linear Unit (ReLU) 20

2.5.4 Fully Connected or Dense Layer $\quad 21$

2.5.5 Stacking the Layers 21

2.6 Transfer Learning 23

2.7 Deep Fashion Dataset $\quad 24$

2.7.1 Identifying Categories $\quad 24$

2.8 Vgg16 27

2.9 Capstone 28

2.9.1 Kratos Project $\quad 28$

2.9.2 Challenges 28

3. DESIGN

$\begin{array}{ll}3.1 \text { Data Pipeline } & 30\end{array}$

$\begin{array}{ll}3.2 \text { Model Architecture } & 31\end{array}$

4. METHODOLOGY

5. RESULTS

$\begin{array}{ll}5.1 \text { Training Loss } & 37\end{array}$

5.2 Top 1 Accuracy 39

5.3 Top 5 Accuracy $\quad 41$

5.4 Model Predictions $\quad 44$

5.5 Source(s) of Error $\quad 46$

6. CONCLUSION 46

$\begin{array}{ll}\text { 7. FUTURE WORK } & 48\end{array}$

$\begin{array}{lr}\text { 8. KEYWORDS } & 48\end{array}$ 


\section{INTRODUCTION}

The human brain is one of the greatest inspirations for the means of computer innovation. The way the neurons fire in a mass frenzy as thoughts are formed is simply incredible. "Humans are exposed to [a] myriad of sensory data received every second of the day and are somehow able to capture critical aspects of this data in a way that allows for its future use in a concise manner." [1] As human beings, we are able to process, learn and adapt to so much, so quickly. The idea of replicating these human abilities and capabilities for learning and adaptation in the form of software is simply incredible. It even sounds too good to be true. To some aspect, it is, but progress has already begun, at least in its early stages. [2]

The potential applications of machine learning for image recognition and prediction are ever growing. [2] The case that many have tended to lean towards have been using machine learning to determine medical diagnostics. [2-4] Even right now, neural networks are becoming more and more commonly used. Some notable examples of neural networks in development are facial recognition, medical diagnostics, clothing recognition or even self-driving cars. [3-8] Machine learning has become more and more prevalent in today's modern world. Technology is always improving, and machine learning is a field blooming with possibilities.

In this thesis, I develop two convolutional neural network models implemented with Tensorflow [21] and train them on the Deep Fashion dataset [8] to identify various categories of clothing. The first model is trained only using the Deep Fashion dataset. The second model incorporates transfer learning by using pre-trained weights from Oxford's Vgg 16 model. Transfer learning is a technique that takes the learned weights of one model and uses these weights to 
initialize another model, which is then trained on a different dataset. My work evaluates the effectiveness of transfer learning and how it may potentially improve on metrics such as accuracies and loss in model development. I believe that that transfer learning will result in higher accuracies, as well as lower training loss than models trained from scratch. Throughout this thesis, I elaborate on development, experimentation, and analysis of model metrics before arriving at a conclusion on the effectiveness of transfer learning.

\section{BACKGROUND}

\subsection{Input and Output}

The input to machine learning computer vision models is generally a series of images that are seen as multidimensional arrays or matrices of different color spectrums. Generally, these input matrices contain pixel values that are scaled by dividing by 255 . This is to accommodate the red, green, and blue color spectrum and simplify computation.

The output of a machine learning model layer is generally another matrix of values. However, the final output of a model is special. It is a prediction on the contents of the input data in the form of a vector of values containing probabilities on the likelihood of specific categories. This is the model's expected result and the dimension of the vector is equivalent to the total number of possible categories.

\subsection{Training and Loss}

For supervised learning, training refers to running inputs through the model with a specific label to identify the category of each output. The data being passed through the model is 
called training data and it consists of a large partition from the total dataset. In fact, datasets for machine learning are generally divided into three partitions, the training, validation, and testing sets. Training sets contain the majority of dataset and the key to them is to be repeatedly passed through the model to train it. Every training example consists of an input and a label for what the correct output should be.

For computer vision and image recognition, training is conducted by passing all the images of the training set through the model and comparing the model's results to that of the correct classification of that data. Going through every image in the training set is referred to as an epoch of training. During each epoch, the model identifies where it has made mistakes and backpropagates the error through the network to update its weights.

Once the weights have been updated for the specific epoch, the model is trained again on the training set to recognize the images. There are cases where if not enough data is presented; the model can actually memorize the order of the images. This is why models may perform really well on their training sets, but fail drastically on their testing sets. This is otherwise referred as "overfitting" to a dataset.

Another key aspect to training is training loss. Training loss is the non-negative penalty value of a bad prediction along the training set. [15] It measures the inconsistency between training labels and predictions. This loss is generally calculated with the mean squared error (MSE), otherwise referred to as the squared difference between the actual value and the predicted value. [16][17][19] Note that a loss value of zero means a perfect prediction is made on individual values. This means that the smaller the loss values become over individual epochs, the better a model becomes at making predictions. 


\subsection{Evaluation}

There are various ways to evaluating a machine learning model. Generally, models are evaluated based on the accuracy of their predictions on the testing set. This is also known as top 1 accuracy. Now, predictions of a model are determined by identifying the greatest probability value of the model's final outputs. This probability value is then mapped to its corresponding category value. This process determines the category predicted by the model. Finally, the prediction is compared against the label to see if the input was correctly classified.

The same process is also conducted for evaluating based on the accuracy of the top 5 predictions. This evaluation works in the same way as the previous, however instead of just the first prediction, the model checks its label against the 5 highest predictions of an input. If any of the 5 predictions match the label, then the input is classified correct. This evaluation process is referred to as the top 5 accuracy and provides a margin of error for model predictions. It's exceptionally useful when classifying images that may contain multiple existing categories that aren't officially labeled by the input image. Top 5 accuracy doesn't just improve on accuracy, but also allows the model to generalize more of its predictions.

Lastly, there is the training loss metric, which shows that the model is improving on the training set over each epoch iteration. The key to this is showing how the model's training loss decreases over iteration. The closer the loss is to zero, the better the model becomes at making predictions on the training set. Note that the success of a model on the training set does not always lead to similar success on the testing set. 


\subsection{Neural Networks}

Neural Networks (NN) are computer systems inspired by the brain's biological neural networks. [9] These systems learn by example and with no prior knowledge. These are often used for image recognition. They are constructed of an input layer, one or more hidden layers, and an output layer of nodes.

\subsection{Convolutional Neural Networks}

Convolutional Neural Networks (CNN) are a branch of neural networks specifically designed for multidimensional data. [1] In the case of image recognition, CNN's view images in small sections at a time. They evaluate images piece by piece and identify specific patterns or filters within said images. [18] Every filter that the CNN finds is like a smaller collection of pixels that the model is looking to identify.

Now, as a means of explanation, I will reference an example from Brandon Rohrer's online course on deep learning. The following figures are an example of the process that CNN's go through to develop their computer vision and improve on their predictions. As we can see from figure 1, say there is a CNN that is used to identify images of X's and O's. For simplicity, assume that the $\mathrm{CNN}$ will always be passed an image of an $\mathrm{X}$ or an $\mathrm{O}$. We'll note that these images are composed of two-dimensional arrays, or pixelated images. Now, given the exact images in figure 1 , a computer can be made to recognize those patterns as X's or O's.

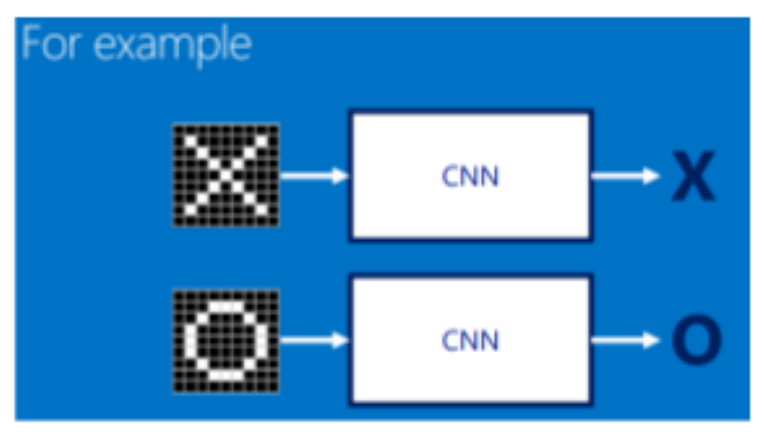

Figure 1: In this example, this is a CNN that recognizes $2 \mathrm{D}$ arrays of pixelated images as X's or O's. [18] 
However, let's say we introduce alterations to our examples of X's and O's. As shown in figure 2, we come upon that exact situation. Our $\mathrm{CNN}$ from figure 1 is now being fed multiple variations of images that are identified as X's or O's. Referencing the X's and O's in figure 1, we

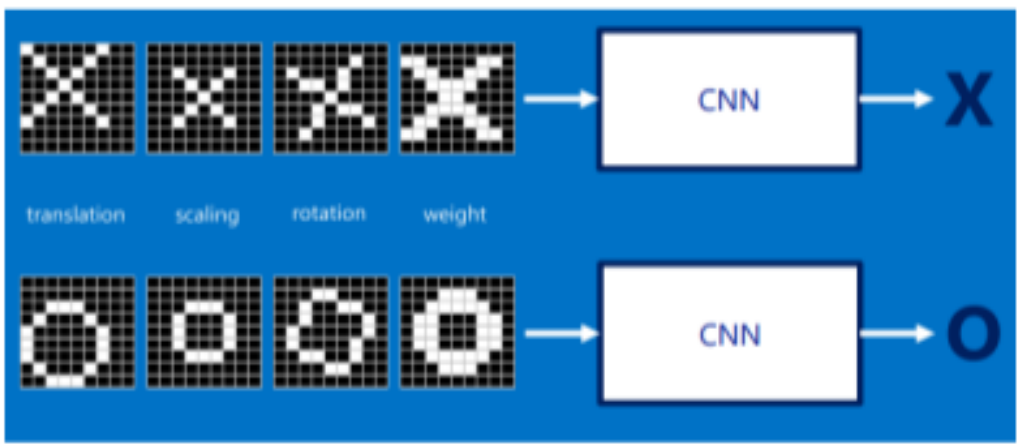

Figure 2: There are a multitude of cases that are considered as X's or O's. The CNN needs to be able to adapt to these images and identify them accordingly. As shown in the slide, the categories have to deal with translation, scaling, rotation, and/or weight. All of these are still classified as X's or O's, but to a computer may be seen as different from the initial pattern. [18] will notice how the images in figure 2 don't match the original pixelated $\mathrm{X}$ and O pattern. The X's and O's in figure 2 have been subjected to slight modifications such as translation, scaling, rotation, and weight. For the human eye, we can still clearly identify these variations in the X's and O's.

However, there is difficulty for computers to classify these images due to their hyper literal nature. All the variations of X's and O's in figure 2, technically, don't match to the original $\mathrm{X}$ and $\mathrm{O}$ presented in figure 1 . This inability to match could be due to even a single pixel being out of place.

To continue on with a computer's hyper

literal nature, figure 3 takes a closer look at the original $\mathrm{X}$ image from figure 1 and the $\mathrm{X}$ rotation from figure 2. It specifically illustrates how the two 2D arrays aren't an exact match. However, it also shows how there are sub-images or filters that are shared among the two and how each of the 2D arrays

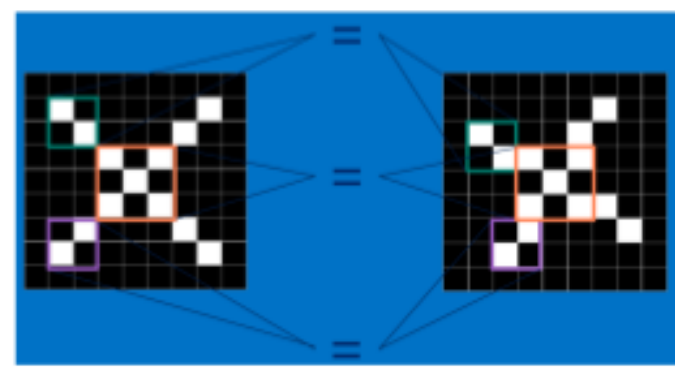

Figure 3: To manage identifying the images that have been slightly altered, the CNN goes about identifying subtle qualities of the classification. In the image to the left, notice the colored boxes in each of the 2D $\mathrm{X}$ arrays. These boxes are the CNN viewing the image in sub-images and identifying filters. [18] 
have specific patterns in common. These similarities are the topic of figure 4, which focuses on those filters. In figure 4, the CNN identifies three specific filters that are unique to the $\mathrm{X}$

Features match pieces of the image

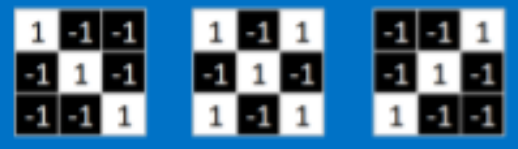

Figure 4: Here, the CNN has identified three filters that are associated with the $\mathrm{X}$ classification. Each of these sub-images are of the dimension $3 \times 3$ pixels. Starting from the left, the $\mathrm{CNN}$ has discovered a diagonal line from the top left to the bottom right. In the middle, there is an $\mathrm{X}$ or crossing pattern. Lastly, it found a diagonal, from top right corner to bottom left corner. [18] category. Starting from the left, these filters are a diagonal

line tilting downwards to the right, the crossing intersection of the X shape, and another diagonal tilting in the opposite direction.

Then the CNN takes those filters and applies them to the input image in an extensive search. The search is conducted by shifting the filters across the image, pixel chunk by pixel chunk, until it finds a matching pattern or completes its search on the entire image. As shown by figure 5, the $\mathrm{CNN}$ goes about searching the full image for each of those sub-images or

filters. In figure 5, the CNN conducts the exhaustive search over the image and finds each of the sub-images throughout its search.

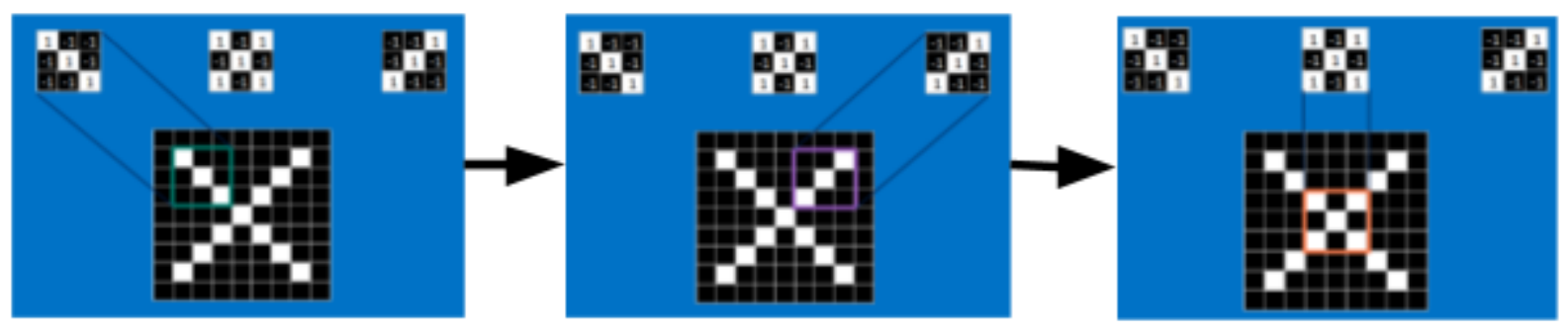

Figure 5: Now, we see how the CNN looks at the big picture and works its way through to identify where each of the filters are in the image. Starting from the top left corner, the CNN identifies the first diagonal of the $\mathrm{X}$, then the right as it continues to view $3 \times 3$ sub-images along its way through the full $2 \mathrm{D}$ array. Eventually the $\mathrm{CNN}$ identifies the crossing pattern in the image and continues along its way as it finds the bottom two legs of the X. Notice how each filter is matched to certain sections throughout the image. [18] 


\subsubsection{Filters and Convolutional Layers}

Convolutional Neural Networks don’t

inherently match filters as immediately as the human eye. They use calculations to match the filters across the whole image. The math used in this process is convolution, hence where CNN's get their name. For the CNN, it doesn't know where the filters match; it computes its calculations in every possible position. As shown in figure 6 , the $\mathrm{CNN}$ searches every $3 \times 3$ area of

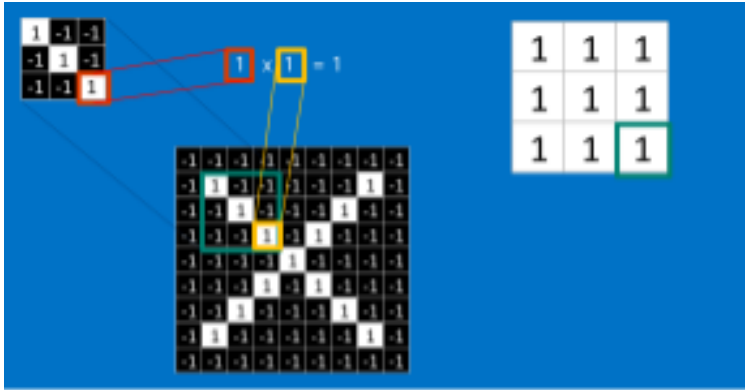

Figure 6: Here, the CNN finds a matching filter and pattern in the image. As shown by the red and yellow boxes, the red is the corresponding filter pixel. The yellow is the corresponding image pixel. Both of those values are multiplied together to get the window in the top right corner. [18]

pixel on the original image with the tilting right filter. Then, once the $\mathrm{CNN}$ identifies a match to the filter, it multiplying each corresponding image pixel by its corresponding filter pixel. The outputs of these element wise multiplications are placed in the $3 \times 3$ white square shown in figure 6 and 7. These outputs are then all summed together. This is done for every position throughout

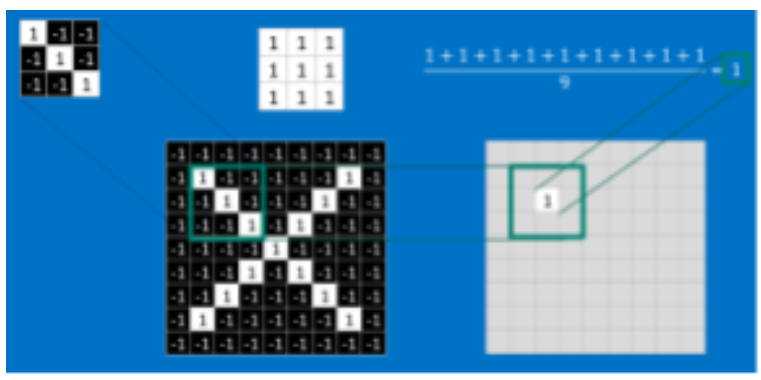

Figure 7: The next step is taking the $3 \times 3$ window in the top center and taking its mean as shown in the top right corner. This results in a 1 meaning that the pattern is a $100 \%$ match at that position. [18]

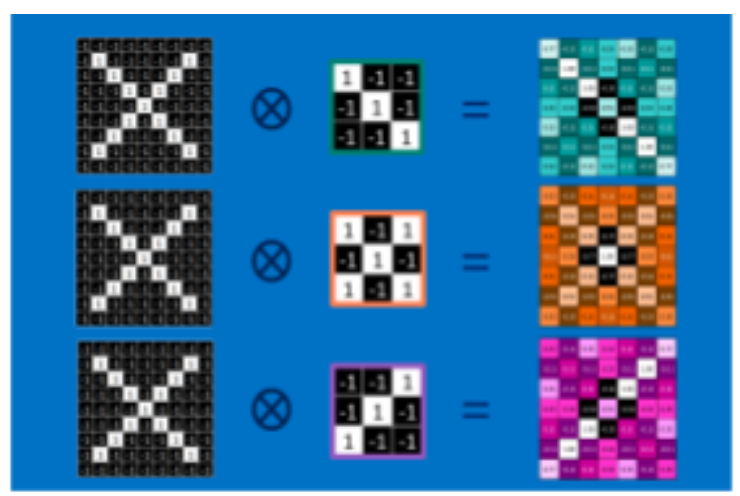

Figure 8: The convolution process is applied to each of the filters on the same image. Their resulting matrices are displayed to the right side of the image. As we can see from the image, the filters are projected into their outputs with the diagonal lines, then the cross section. [18] 
the full input image. In addition, as seen in figure 7, this process condenses the image down to the more essential aspects of the matching filter.

Once through the convolutions, figure 8 shows the completed output for each of the three filters from earlier. Something to note is the original input was a 9x9 pixel image of an $\mathrm{X}$. Then, after convolution, the output becomes a $7 \times 7$ pixel image of averages that highlight the key aspects to each of the $3 \times 3$ pixel filters.

\subsubsection{Pooling Layer}

The max pooling layer is a means of converting larger images into smaller ones, "while preserving the most important information in them."[18] This process is

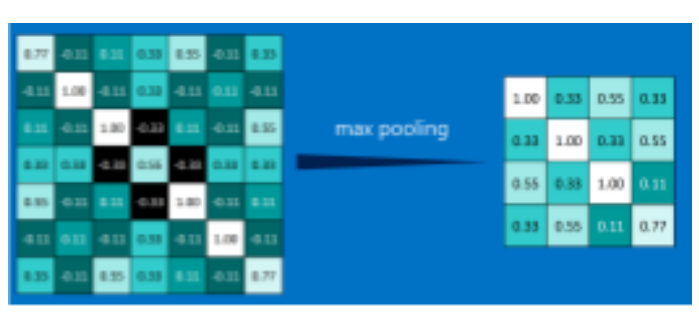

Figure 10: Once the max pooling is complete, its output is approximate a quarter the size of the initial input. Note how the key features of the image are still displayed, the diagonal line declining towards the right. [18] conducted by moving

across the image in

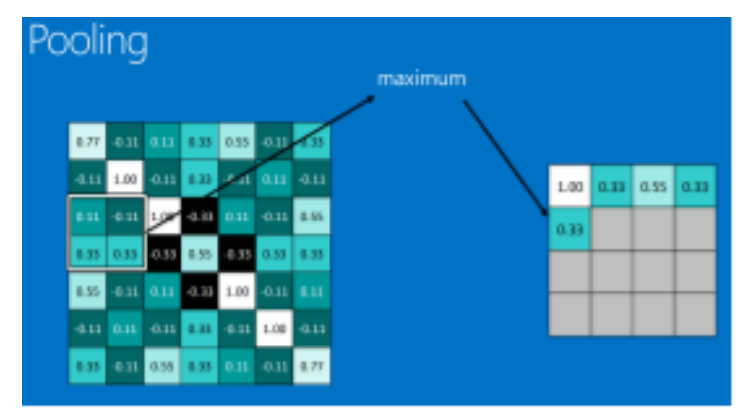

Figure 9: The $\mathrm{CNN}$ takes a window of $2 \times 2$ and slides it across the input matrix. Notice how the output is being populated with the maximums of each $2 \times 2$ slide window. This is to preserve the most important aspect of the initial image. [18]

small fixed sized windows and taking the maximum from each of those windows. Generally, in implementation, a window size of $2 \times 2$ or $3 \times 3$ pixels with a step size of two perform rather well. This process is illustrated in figure 9 with a window of $2 \times 2$ and step size of two.

Once the pooling process is complete, the image is about one fourth the size of the original image, as shown in figure 10 . Note, because the pooling layer took the

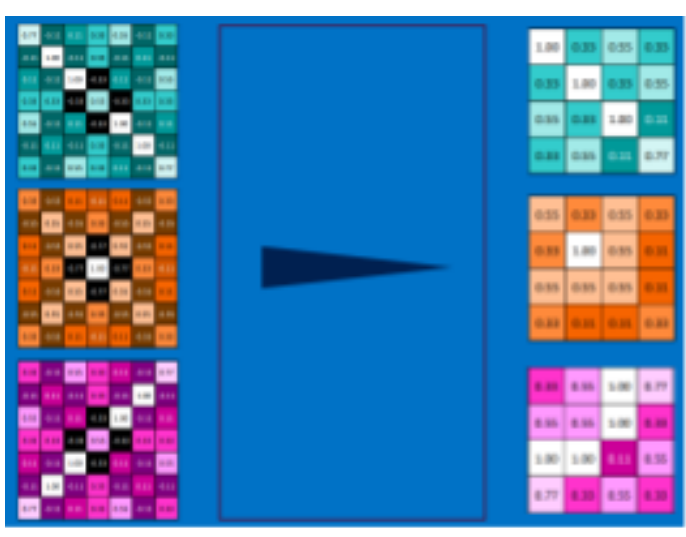

Figure 11: Here, we see the result of each filter after we apply the max pooling layer. For the most part, the patterns are more generalized in there simpler forms. This will help keep the computer generalize and be less literal in its analysis of images. [18] 
maximum of each step, "it preserves the best fits of each filter within the window."[18] This actually helps keep the computer from being so literal because the simplification means a filter need only fit within the window to match. Hence, the process also helps minimize mass computations.

Then as shown in figure 11, these pooling layers are applied for each filter. The outputs of the pooling layer are much more simplified. Yet, for each of the three sub-images, the key aspects to each filter are still represented among the quarter sized outputs.

\subsubsection{Normalization with Rectified Linear Unit (ReLU)}

A Rectified Linear Unit (ReLU) replaces all negative numbers with zeros. As simple as it may seem, this layer plays quite a significant role by keeping the learned values from approaching infinity or getting too close to zero. This keeps the computation from getting out of control. As shown in figure 12, this layer goes from pixel to pixel to replace all negative values.

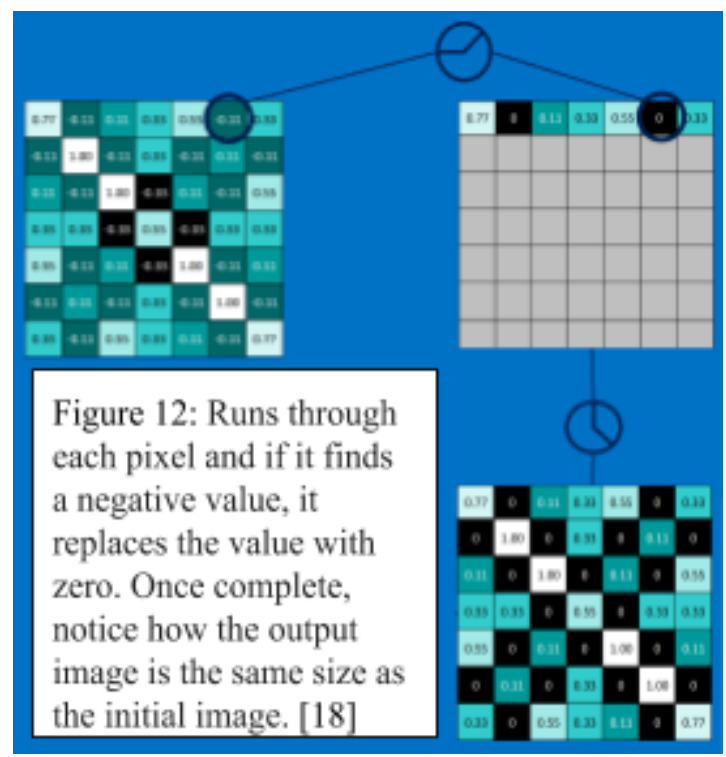

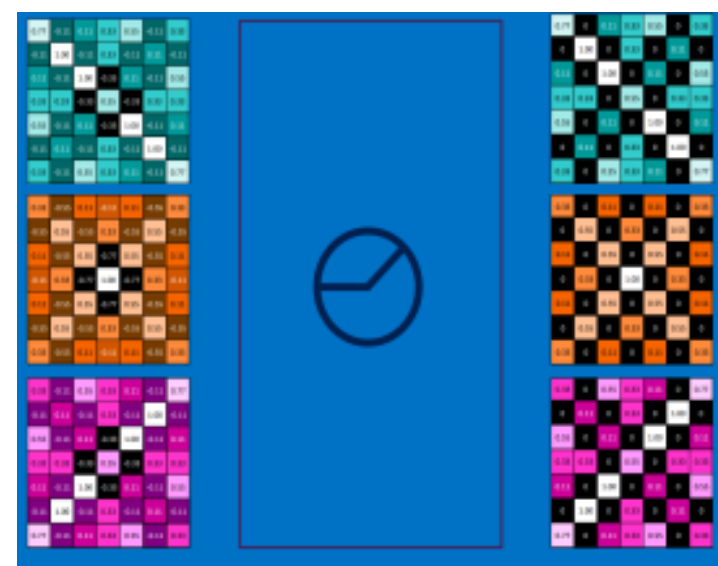

Figure 13: The ReLU is applied to each of the filters to prevent values from approaching infinity and therefore allowing easier computation. [18]
Figure 12 also shows the

completion of the ReLU layer towards the bottom right

corner. Something to notice is how the output image persists

with the same dimensions as the input image. It's just

without negative values.

Figure 13 shows how the ReLU layer is applied to each of the filters found before. Replacing all negative values 
significantly reduced the amount of computation required for each filter. Another key aspect to notice is how the key aspects of the filters have been enhanced in the output image.

\subsubsection{Fully Connected or Dense Layer}

The fully connected layer or dense layer converts the matrices input into a singular, linear list. This is shown in figure 14 where the three, $2 \times 2$, matrices are converted into a one dimensional list of $1 \times 12$. From here, every input is connected to every output via some assigned weight value. The weights of the list are then polled to determine the output. This layer is best used for classification, otherwise referred to as prediction when it is the final layer. This can be shown in figure 15 where polling the layer determines the image to be classified as an $\mathrm{X}$.

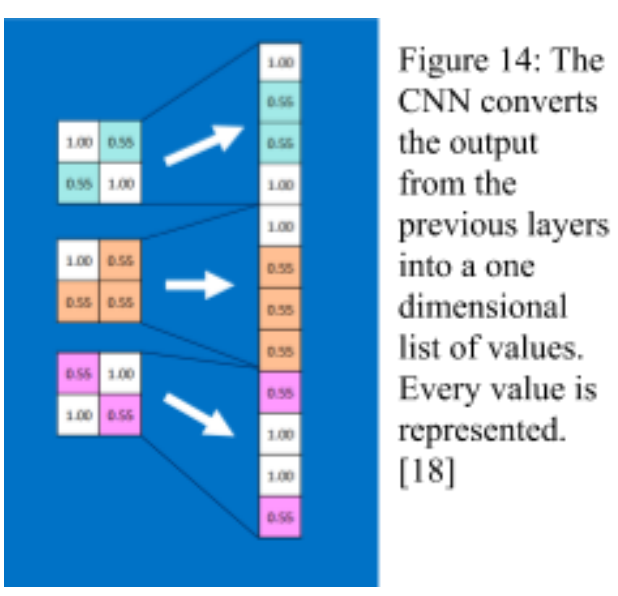

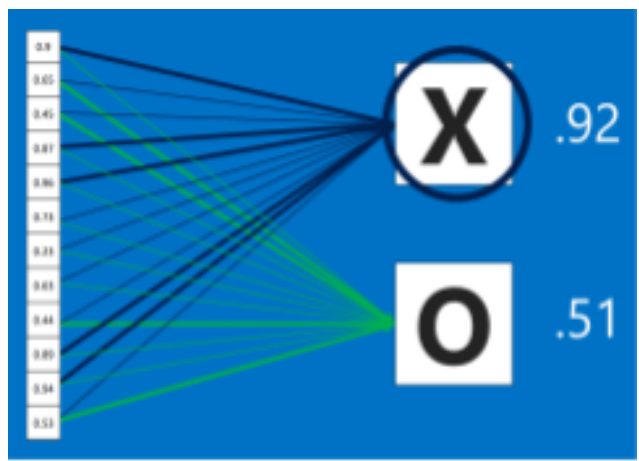

Figure 15: The 1D list of 12 values then is polled to determine if the image the $\mathrm{CNN}$ was passed is an $\mathrm{X}$ or an $\mathrm{O} .[18]$

\subsubsection{Stacking the Layers}

Finally, it comes to putting all of the layers together. For each of the layers shown

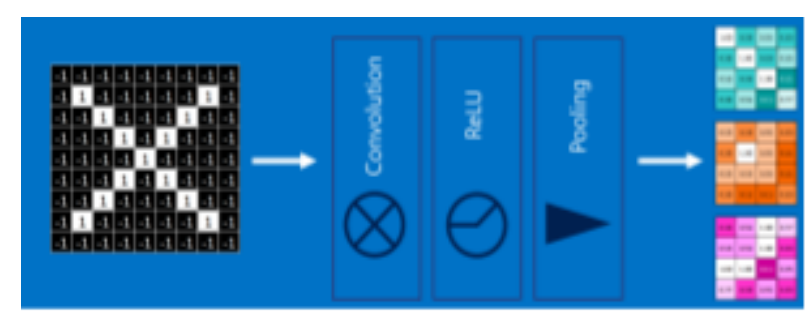

Figure 16: The outputs of one layer are continually passed to the next layer as input. [18] throughout this example, the output of one layer has becomes the input of the next one. In figure 16, it shows how the original pixel input image is fed through a CONV, ReLU, and POOL layer. The 
outputs from these three layers are applied one after another. This process continues until it reaches its final output layers in which the model can then produce a prediction.

This process of stacking layer after layer can be done a multitude of times. In most model architectures, the pattern repeats with a series of CONV's, ReLU's, then POOL layers. Stacking these layers together create a deep model architecture. Deep CNN's follow a hierarchy in which the model can pick out more information from the outputs of every layer. The deeper the model goes, the more layers of abstraction it learns.

Now, as shown in figure 17, this is a typical example of what a deep CNN looks like. Here, the same input image is fed in as shown in the previous figure. The model architecture

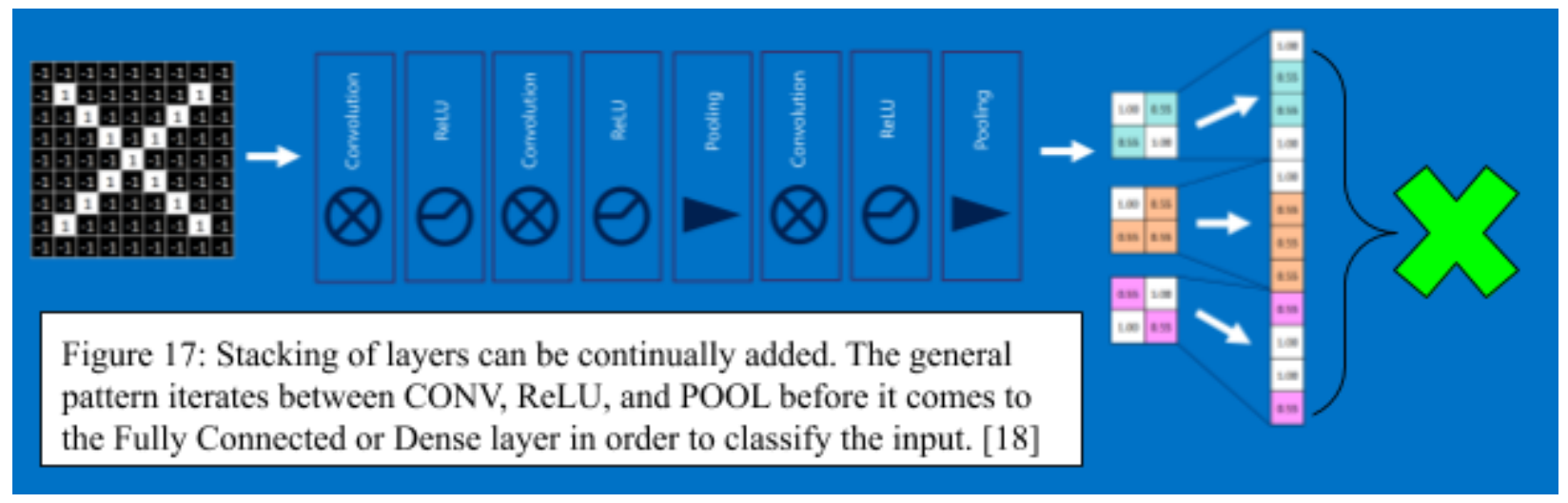

alternates between CONV, ReLU, and POOL layers before it reaches its end. Towards the right, after the final pooling layer of the model architecture, are three matrices. From the example, those matrices are the outputs of the three filters found in the beginning. Now, notice how those matrices are converted to a linear list. This is the final fully connected layer of the model, where the weight values of each element of that list are polled to determine a prediction on the initial input image. 


\subsection{Transfer Learning}

"Transfer learning is the improvement of learning in a new task through the transfer of knowledge from a related task that has already been learned." [10] Imagine it as having a model with basic knowledge, then continuing to train from that point on. Generally, transfer learning helps by passing on the similarities of the tasks such as edge detection or certain pattern recognition.

The hypothesis I'm exploring is that transfer learning will result in better metrics than a model without transfer learning. As presented in the figure 18 below, the expectations are

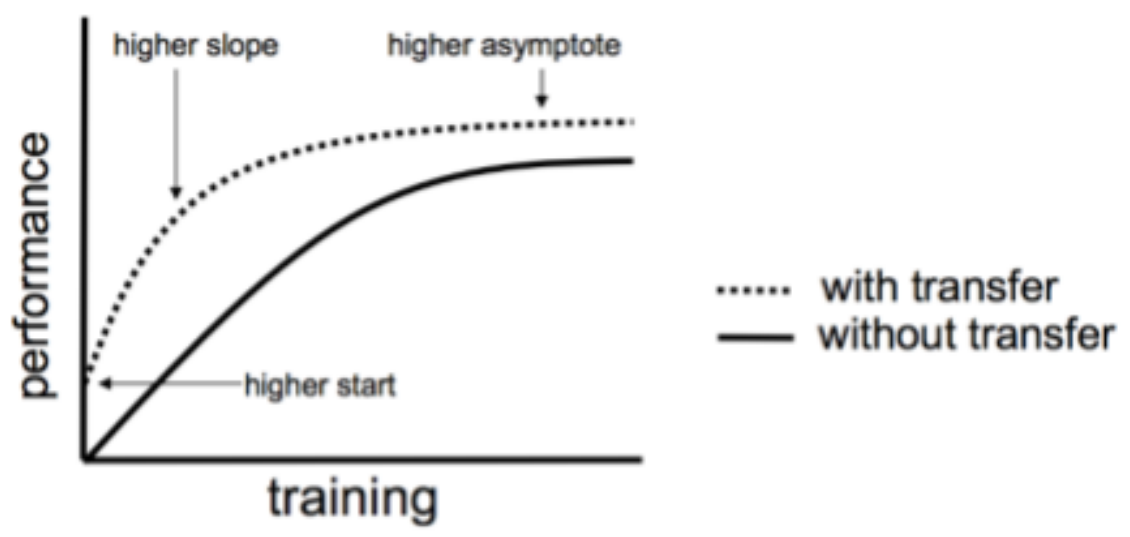

Figure 18: "Three ways in which transfer might improve learning." [10]

improved initial performance, time to learn, and final performance. [10] Notice the significant difference between the performance of a model using transfer learning, and the one without.

Now, in the form of implementation, transfer learning is slicing off the final output layers of a pre-trained model and replacing them with output layers that are specific for the new target output. In my implementation, the pre-trained model is Oxford's renowned Visual Geometry Group, also referred to as Vgg16. The Vgg16 model is the second place runner up of the 
ImageNet competition of 2014. [11-14] It was pre-trained on the ImageNet dataset to identify images into 1000 potential categories of classification. Also, due to the model's impressive performance on the ImageNet data, I believe that using Vgg16 for transfer learning could pass on its success to new transfer learning models.

\subsection{Deep Fashion Dataset}

Deep Fashion [8] is a large-scale clothing database containing images of varying qualities. Image quality ranges from everyday consumer camera quality to professionally taken photos. The database also has documentation annotating which images belong to which category. All images in the dataset are labeled with 50 categories and 1000 different clothing attributes. This dataset contains 289,222 images of clothing. For this thesis, the focus will be on the 50 categories of clothing.

\subsubsection{Identifying Categories}

The categories classification section of the Deep Fashion dataset contains 50 different categories which can be found in Deep Fashion’s 'list_category_cloth.txt` file or in the code section specified below. 


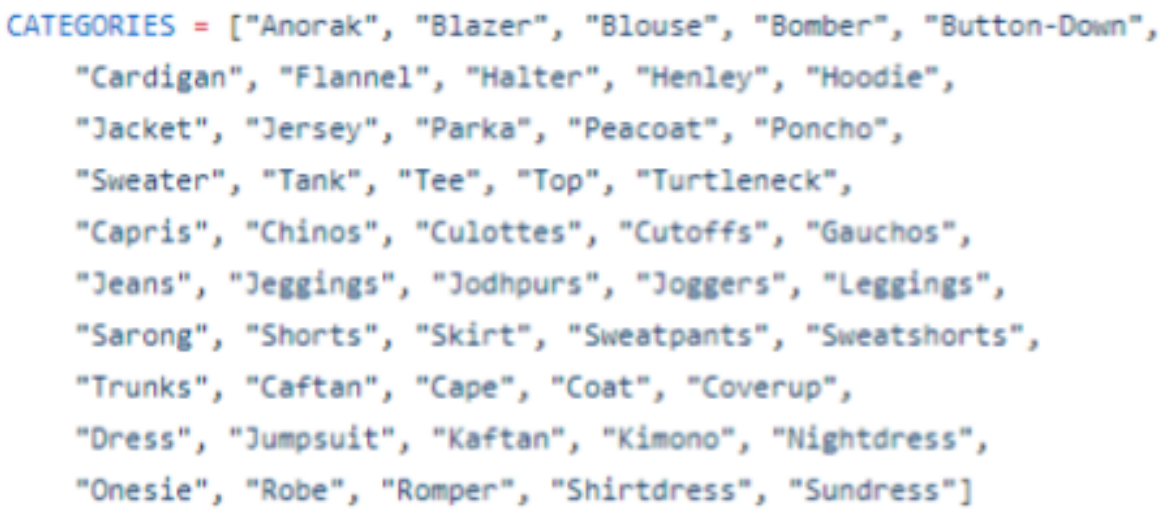

Figure 19: All fifty categories of clothing from the Deep Fashion Category Dataset. [8]

To go along with these 50 clothing categories, the dataset has 289,222 varying sized images containing instances of the 50 different clothing categories to identify. In the Deep Fashion documentation, there are text files containing the means of splitting the data into training, validation, and testing sets. 40,000 images belonged to the test set, 40,000 for the validation set, and the remaining 209,222 of which are for the training set. Also, for each of these images, Deep Fashion has a 'list_category_img.txt` file which contains the actual labeled information for each of those 289,222 images. In short, it specifies what category of clothing the images are classified under. 
Figure 20: Representative images from the Deep Fashion Category Dataset[8]. These images are all varying examples from the Blouse category (Category_label 3).
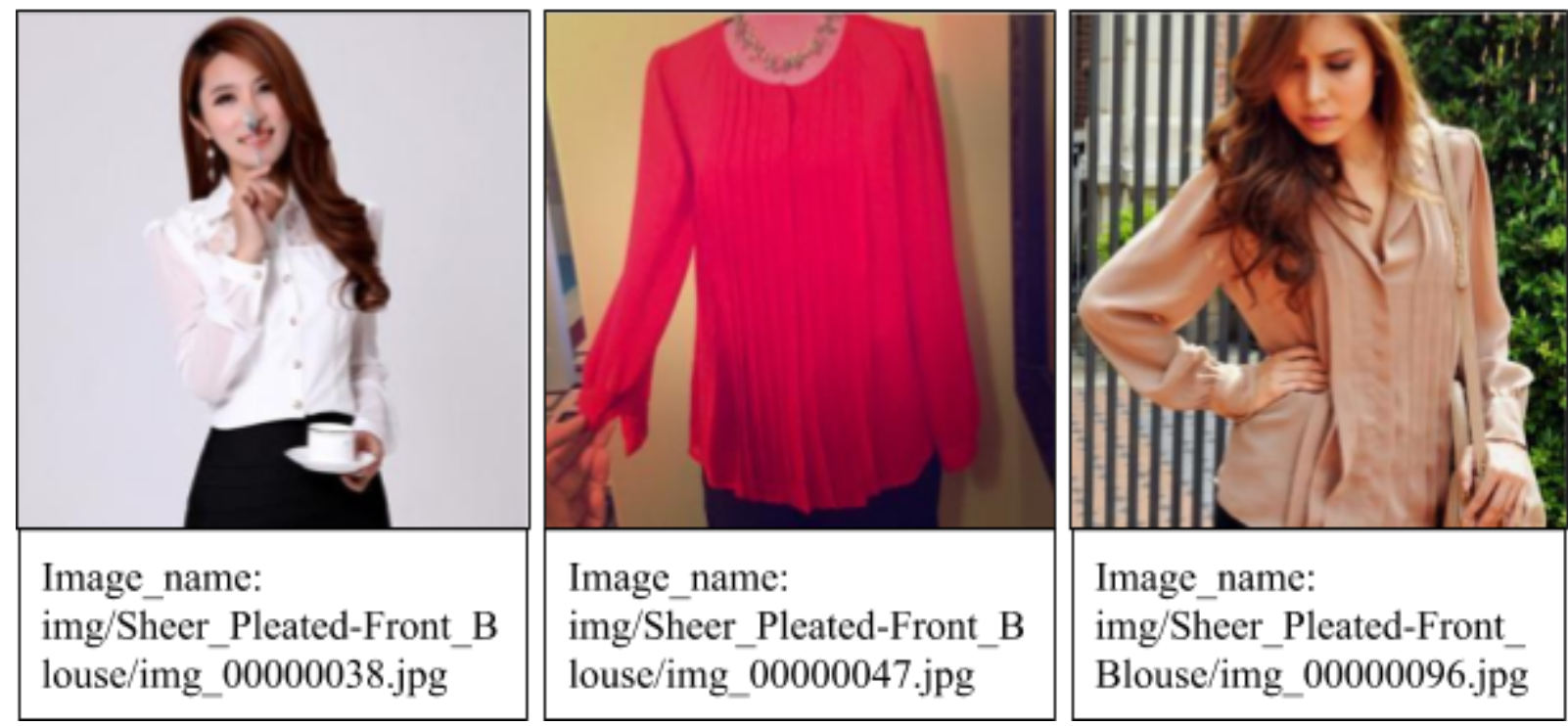

In figure 20 above, there are a few example images from the blouse category. As shown in the example images, the blouses have different colors, poses, and photo quality. Clearly, all the images are qualified as category 3, a blouse. In human eyes, the clothing in the images isn't a problem to identify. However, for computer vision, the difficulty lies within the diversity of the dataset and those images. Specifying on the images, the background, the individuals in them, the colors, or even the photo quality is enough to alter a model's predictions on the image.

With the difficulties in datasets, if the data is unbalanced, the model will learn to represent that bias. For the Deep Fashion dataset, the categories section does have a slightly greater skew towards "leggings" and "skirts". However, it also has certain limitations in its representation of categories like "kimono" and "Caftan". In short, computer vision is fairly sensitive to the way the data is represented and distributed. 


\subsection{Vgg16}

As previously mentioned, Vgg16 is a machine learning model developed by Oxford's Visual Geometry Group for the 2014 ImageNet competition. It is trained on the ImageNet dataset to identify images into 1000 distinct categories. Under the hood, Vgg16 is a Convolutional Neural Network composed of 19 layers not including the input and output layers. As shown in

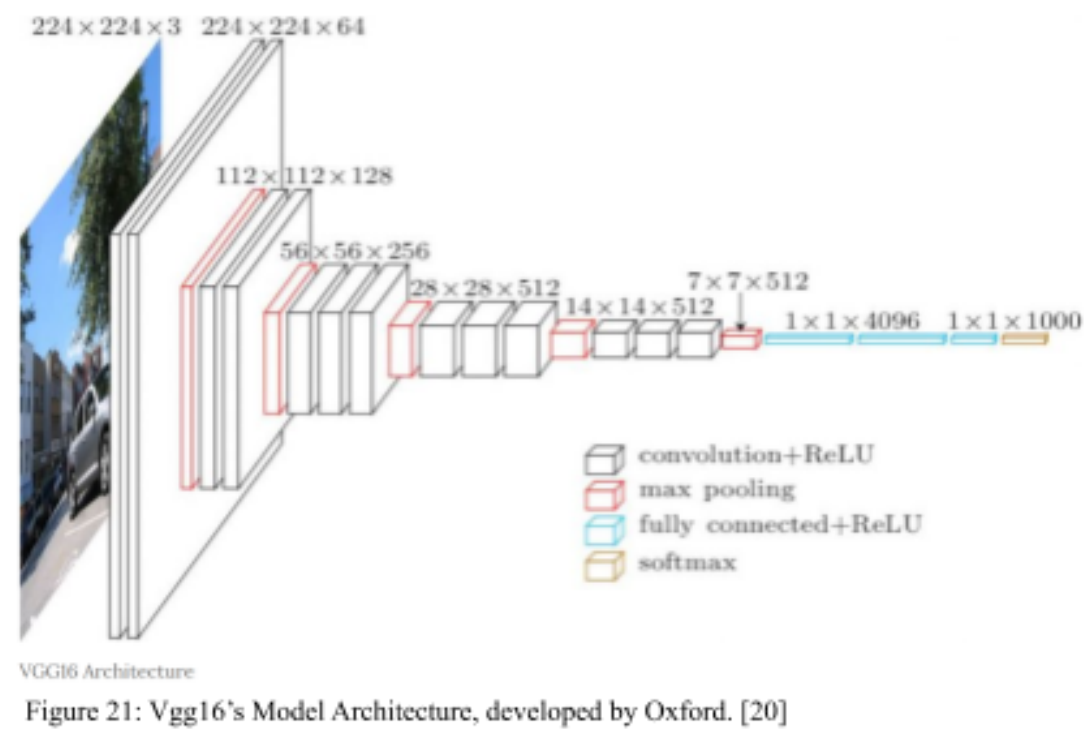

figure 21, this is a typical example of a CNN. The layers are stacked on one another and the inputs of one layer go on to become the inputs of another [18]. At the start, the model takes in an RGB image of $224 \times 224$. The image is passed into two convolutional layers, max pooled, two convs, another pooling, then three convs, a pooling, three more convs, pooling, three convs, a final pooling, then a fully connected layer followed by a softmax layer of 1000 . This final output layer is set to 1000 to represent the 1000 distinct categories of the ImageNet dataset. Note this is the model architecture I will use to conduct transfer learning. 


\subsection{Capstone}

The Computer Science Capstone spans over two-terms. It is where teams of students receive the opportunity to simulate real-world working experience and work together to develop an open source product/solution for an issue proposed by an outside sponsor. This thesis is being done in unison with the capstone project, Kratos. Kratos contains the source code to the training and creation of machine learning models that can identify images of clothing for the sponsor, Nike.

\subsubsection{Kratos Project}

Kratos is an open source AI system developed to identify images of clothing. The Kratos Project was initiated at the start of Fall term 2018 in the first section of the Computer Science Capstone. This AI System contains multiple machine learning models that have been trained on the Deep Fashion dataset to identify different factors of clothing images. The specific task of Kratos is to identify the categories, colors, and attributes of clothing. Implementation wise, it is composed of three category models, two attribute models, and one color model. Full documentation for set up, usage, and modification are specified on the Kratos-ai github.

\subsubsection{Challenges}

There were multiple and various resource limitations. The first of those major limitations was trying to develop machine learning models on my outdated, non-GPU, laggy laptop. My computer repeatedly crashed whenever trying to run or compile the tensorflow models. Loading the dataset itself, on low-quality, took 14 hours on my computer. The processes took up the 
whole CPU. In turn, meaning that my computer was a vegetable while I waited for the model to eventually compile and crash the computer. So developing on my local device's systems were not an option.

The second challenge was after switching to development on the school Linux servers. I continually ran out of memory. There is a strict limitation on individual memory use. Limitations on the school Linux servers really hindered my progress because I couldn't save my work if my model saved first. Meaning I had to choose between saving the work I've been doing for the last few hours, or saving the results I've been waiting on for twice the time. Not a choice I was eager to make. Eventually, there was a meeting to address this space issue for everyone. Our team lead acquired a $100 \mathrm{~GB}$ stash to be created just for the Kratos team. So that solved the dataset and space issue.

Third, varying environments and versioning issues among team members. Trying to make code run on one machine is bad enough, trying to make it run on everyone else's is a different story. Eventually, the team settled on a uniform environment for development. Everyone needed to set their environments accordingly.

Fourth, training on the Deep Fashion Dataset with only CPU's took hours (for one epoch). Eventually, we were given access to Nvidia-GPU 9's. The following issue was that the Nvidia-GPU's didn't run with the version of tensorflow we had. Eventually, I had to downgrade to tensorflow-gpu 1.9.0. after the completion of the Kratos project. 


\section{DESIGN}

The development process starts with creating a data pipeline to read in all the Deep Fashion images with the correct category labels. Those images are then converted into RGB pixel images. After the image conversion, the data is split into three sets for training, validation, an testing. Next, the images for each set are piped into the model architectures for training. The metric data from each of those sets is then recorded into separate .csv files, in between every epoch iteration, and are later used for model analysis.

\subsection{Data Pipeline}

The data pipeline is a crucial piece to any machine learning model. It separates out the training, validation, and testing datasets with their corresponding labels. Each of those datasets contains hundreds if not thousands of images to be fed to the model for training or prediction. Deep Fashion's Category datasets contain images of varying sizes. For this model, the images need to be of the same size and dimensions. That means that the images first need to be preprocessed, read in as RGB, and resized. Now, preprocessing needs to be done to all images that this model takes. Knowing such, brings to light the matter of the image's height and width ratio. To accommodate for that, cropping and padding of the image are done to properly adjust the image to the specified dimensions.

Other aspects that need to be accounted for are equal distribution among the 50 categories, order randomization, and the inclusion of noise or other factors of image skewing. Equal distribution and data shuffling are important to help prevent model overfitting. For 
example, if the data is solely from the class "leggings", the model will learn to identify everything as "leggings" and its accuracy on the training data will skyrocket, but the accuracy on test data will drop significantly. The reason for randomization is to keep the model from memorizing the order of the images and then applying that knowledge to the test set. Lastly, the noise factor is to help the model generalize and deal with perhaps images of varying sizes or backgrounds. Most of the time, pictures taken in the real world aren't the same as those from the training set.

\subsection{Model Architecture}

As described by the Torrey and Shavlik, in their work with transfer learning, transfer learning is advantageous due to its improvement in starting accuracy, rate of improvement, and overall maximum accuracy. [10] To verify the truth to this theory, two model architectures are developed to train on the Deep Fashion Category dataset. These models will both contain the same input and output layers to identify the fifty categories of clothing images. Their input layers will take in images of $250 \times 250 \times 3$, these being the height, width, and RGB dimensions of the clothing image input. Regarding the output layers of both models, each will contain an ending dense layer of 1000 , then a dense layer of 50 for each of the clothing categories.

Now, before continuing, both models must be named for clarity. The first model will be referred to as the scratch model. This means that the model has received no prior training on any other data and its weights have been recently developed and tuned for the Deep Fashion Category dataset. The model's architecture, aside from the input and output layers, is made specific for Deep Fashion's dataset. 
The second model for this experiment will be called the Vgg16 transfer model. This model, as the name implies will use transfer learning to develop its model. The architecture of the Vgg16 transfer model will use the weights of the pre-trained, ImageNet runner up, Oxford's Vgg16. It's also important to note that Vgg16 was originally trained on the ImageNet dataset to identify images into 1000 different categories. This means that the Vgg16 transfer models weights have already been tuned to the ImageNet dataset, but are now being converted to make predictions on the Deep Fashion dataset. In terms of architecture, the main body of this architecture will be from Vgg16.

Now, in figure 22 and 23 below, the details of both model architectures are exposed. The key to notice in these architectures are how both models conclude with the dense layers of 1000 and 50. These values refer to the final outputs of both models. Given an input image of $250 \times 250 \times 3$, the models will continue to pass the image through layer after layer until it reaches the final output layer in which they spit out the one dimensional list of 50 probabilities. Where each index corresponds to the fifty Deep Fashion Category labels. 
Figure 22: Scratch Model Architecture

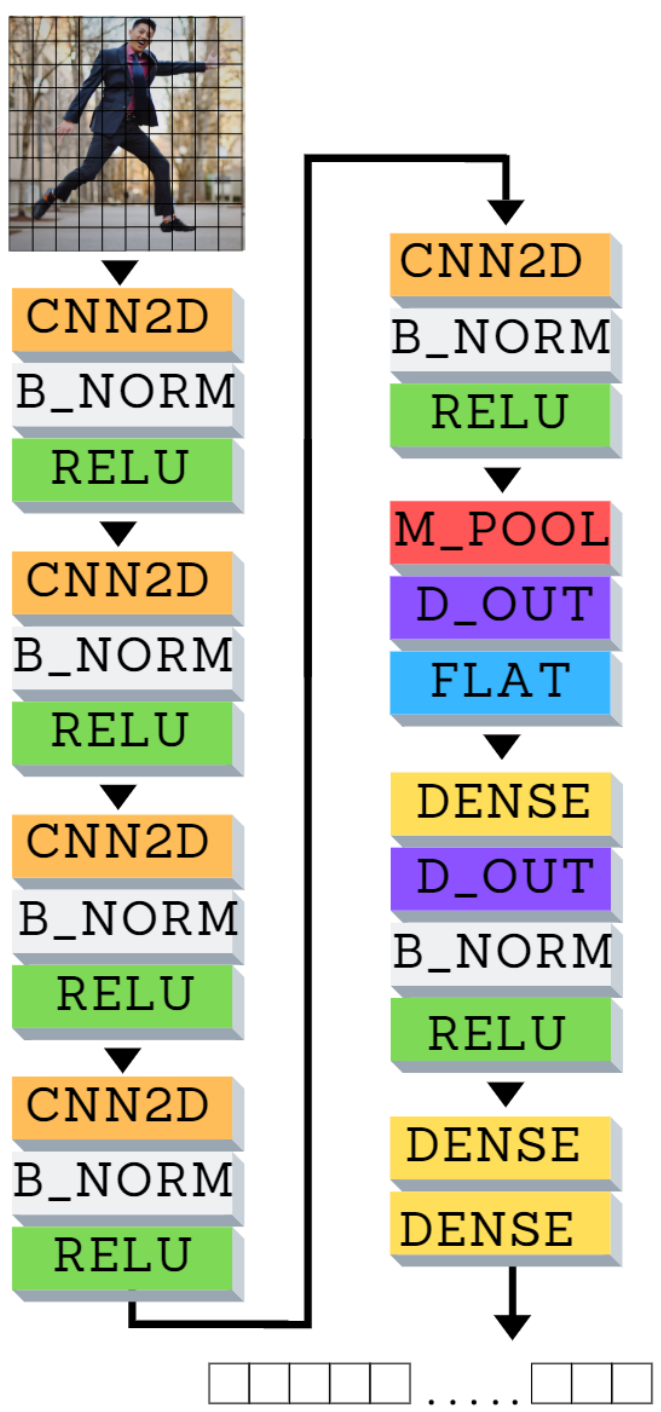

Figure 23: Transfer Model Architecture

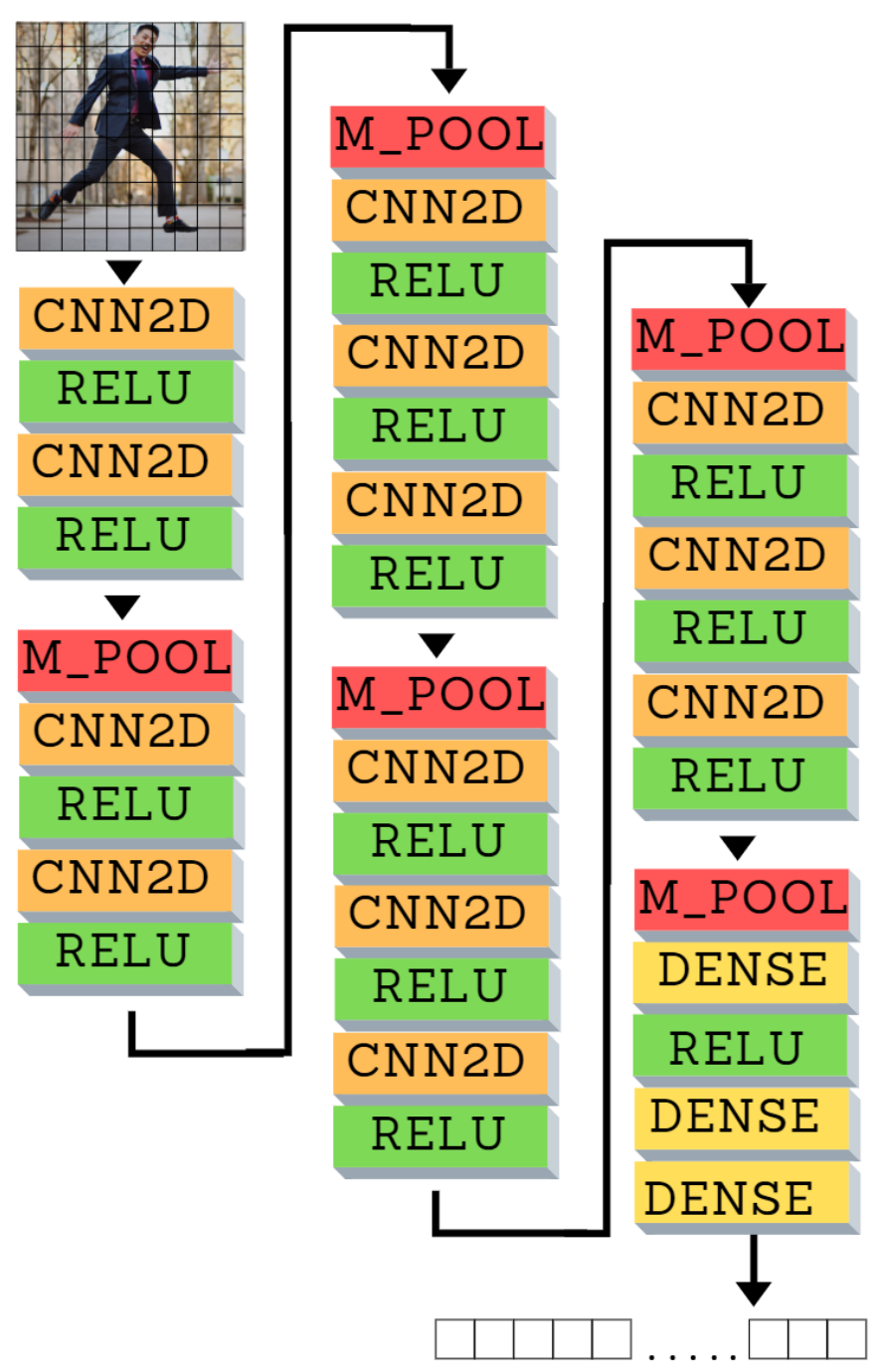

Figure 24: Model Architecture Key

\begin{tabular}{|c|l|}
\hline CNN2D & $\begin{array}{l}\text { = Convolutional Neural } \\
\text { Network 2 Dimensional } \\
\text { Layer } \\
=\text { Rectify Lincar Layer }\end{array}$ \\
FLLU T & $=$ Flatten Layer \\
M_POOL & $=$ Max Pooling Layer \\
\hline
\end{tabular}

\begin{tabular}{|l|l|}
\hline D_OUT & $\begin{array}{l}=\text { Dropout layer } \\
\text { B_NORM } \\
\text { = Batch Normalization } \\
\text { Layer }\end{array}$ \\
\hline DENSE & $=$ Dense Layer \\
\hline|||| $\mid$ & $\begin{array}{l}=\text { Output Layer of size } \\
50\end{array}$ \\
\hline
\end{tabular}




\section{METHODOLOGY}

This section describes the process in which I conducted my experimentation. Before I began experimenting with the Vgg16 model, I downloaded the pre-trained weights from the tensorflow API. After which, I conducted the following.

The first step was creating the two machine learning models specified in the model architecture section. The first model is developed from scratch meaning that the full architecture has been developed just for this dataset and has not been exposed to any previous data, such as the ImageNet datasets. The architecture for this scratch model is made from a convolutional input layer of 250x250x3 followed by a batch normalization and rectify linear layer before passing the output of those layers into four other stack of the convolutional, normalization, and rectify linear layerings.

Then, the result of those layers goes through a pooling and dropout layer of $25 \%$. Next, another dense layer of 2000 with a 50\% drop out rate and rectify activation. Finally, this model's architecture concludes with two final dense layers, dense of 1000, and dense of 50. Note, originally, the fifth layer of the scratch model was originally set to 128 filters. However, due to resource limitations, the layer was modified to contain 80 filters instead of the original 128 filters.

The second model, the Vgg16 transfer model, is set up with the same input and output layers as specified in the scratch model above. However, the Vgg16 transfer model contains 19 layers. Of those 19, I set the first 18 layers to non-trainable to keep the weights of the model intact. The model contains a series of layers as follow: two sets of two conv layers and a pooling 
layer; three conv layers followed with a pooling layer, then concludes with two dense layers. Now of the 19 layers, only the last one is set to trainable. To follow, I then add an additional output layer, specified in the scratch model, as the final layers to the Vgg16 transfer model.

Once both models were created and their model architectures tuned accordingly. I put both models against each other using the images from the Category section of the Deep Fashion Dataset. I then set the parameters in my model_architecture file. Both models would be trained with a batch size of 10 , for a maximum amount of epochs specified at 50. Image height is set to 250 pixels, image width of 250 pixels, and data percentage of 0.05 (5\% of the Deep Fashion Dataset). The purpose of this file is to ensure that the key changing factor in the experiment is the architecture of the scratch and transfer models.

After specifying the parameters for each experiment in the model_architecture files. I run the load_and_train file to initialize a series of csv files to store metric data. At the start of the load_and_train file, it records the metrics into specified csv's for epoch zero. Then conducts its first training before appending more metric data to the specified metric csv's and saving the weights of the model in training. This file then terminates with multiple initialized csv files and a saved weight for one epoch of training. I do this for both models in separate directories to ensure there is no confusion in data.

Next, I open a screen in the linux system to run my processes in the background. This is done to prevent training crashes from a loss of internet. Once in the screen, I run my continue_training script about five times, one after the other, in order to manage any unexpected program terminations during screen experimentation. The continue_training file is designed specifically to pick up from the last saved metric and continues to train the model from that point 
on. Running the continue_training file after the load_and_train file completes allows the model to pick up the saved model and train it for 49 more epochs.

Now, in the case of crashing, assume the model crashes on the 18th epoch. However, it saved the 17th epoch metric. Running the continue_training file again would pick up the crashed training on the 18th epoch and continue to train the model until it reaches 50 recorded metrics. The additional command for continue_training are solely a means of precaution. Repeat this step for both the scratch and the $\operatorname{Vgg} 16$ transfer models.

Once both models have trained for 50 epochs and the continue_training scripts have terminated. Gather the csv's for training loss, top 1 accuracy, and top 5 accuracy. Note that the training loss data belongs to the training set, while top 1 and top 5 accuracy belongs to the testing set. Once again, I am very careful to keep my data in separate directories.

Next, I plot the results of the experiment and label the axises accordingly. The $\mathrm{x}$-axis is always set to the number of epochs and the y-axis is based on the metric being recorded. Repeat this step for both models. Then, after all the metric csv's have been plotted and labeled, the analysis and discussion of model performances can begin.

\section{RESULTS}

After training both the scratch and Vgg16 transfer model on images of 250x250 pixels by 3 color channels, with a batch size of 10 , and data percentage of 5 , for 50 epochs, the graphed metrics are displayed below. Each plot has a discussion of results comparing the scratch model to the Vgg16 transfer model. Note that raw data can be found in the Appendix I Raw Data section. 


\subsection{Training Loss}

\section{Plot 1: Loss on Training Set}

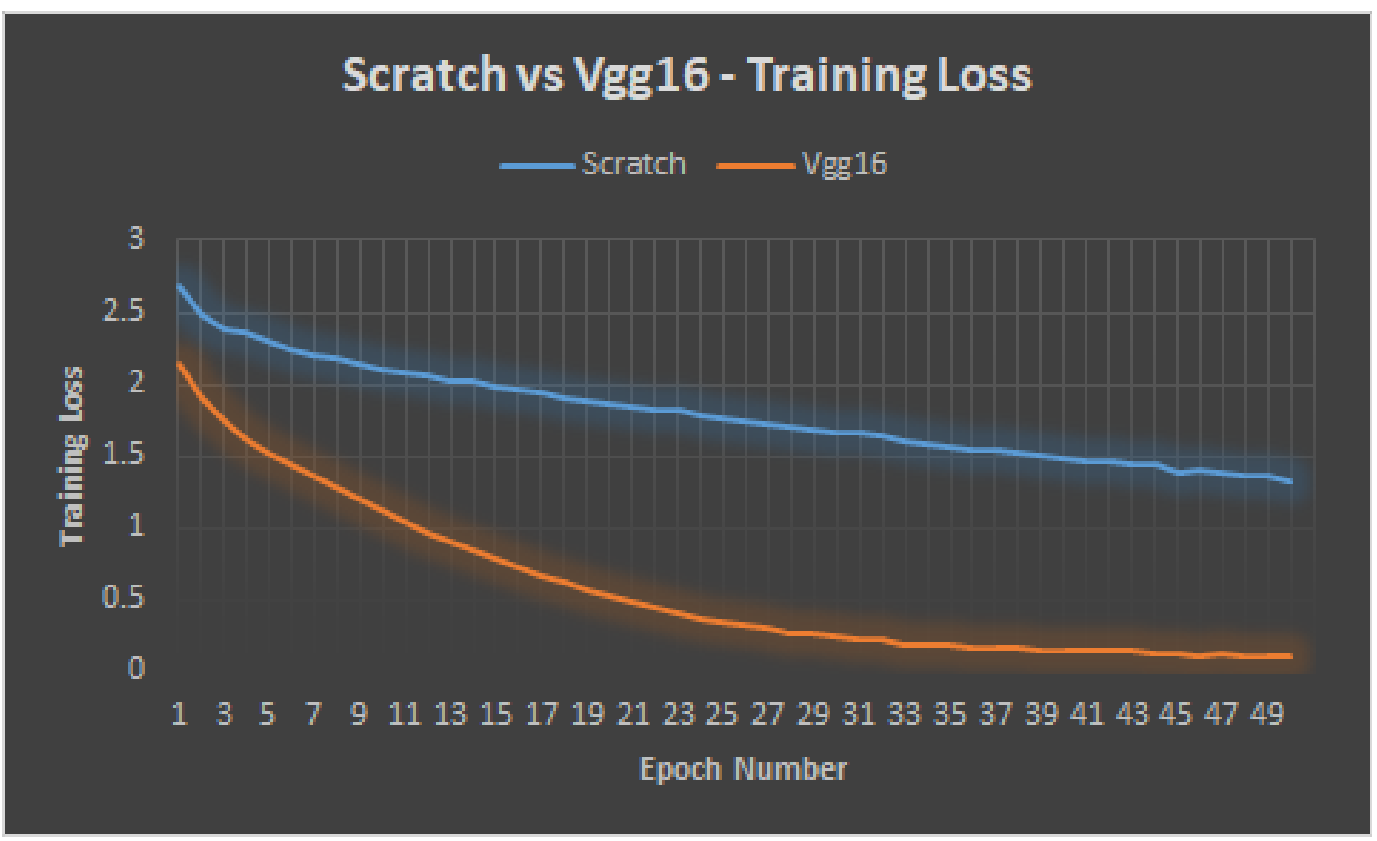

Table 1: Highlights of Loss on training set data.

\begin{tabular}{|l|l|l|l|l|l|l|}
\hline \multicolumn{7}{|c|}{ Training Loss } \\
\hline Epoch \# & 1 & 10 & 20 & 30 & 40 & 50 \\
\hline Scratch & 2.68 & 2.11 & 1.86 & 1.67 & 1.49 & 1.32 \\
\hline Vgg16 & 2.14 & 1.12 & 0.53 & 0.24 & 0.14 & 0.11 \\
\hline
\end{tabular}

As stated in the background section, training loss is the non-negative penalty value for bad predictions throughout the training set. The purpose of the loss metric is to measure the inconsistency between training labels and predictions. The loss in this experiment is calculated with the mean squared error (MSE). This MSE is the squared difference between the data's label and the prediction. This implies a loss of zero is a perfect prediction on the given input. 
Knowing that loss is the metric that we want to minimize, we can look at the data and see that the Vgg16 transfer model starts with a lower loss value than the scratch model. Vgg16 starts with an initial loss of 2.14 and the scratch model has an initial loss of 2.68. Then, as the epochs go on, the Vgg16 transfer model is seen declining at a faster rate than the scratch model until about epoch 29. The scratch model seems to have an almost linear decline in loss, it's good to see the loss value declining, but the transfer model drops at a much more drastic rate and ends with a much lower loss value than the scratch model.

By the 50th epoch, the scratch model comes to a final loss of 1.32 and the Vgg16 transfer model ends at .11 loss. From this, we can see that the Vgg16 model has a loss of 1.21 smaller than the scratch model. Then looking at the overall loss for each model, scratch begins with 2.68 and concludes with 1.32 , resulting in a drop in loss of 1.36. For the Vgg16, it begins with an initial 2.14 loss, then goes to a .11 loss, meaning the transfer model had a drop in loss of 2.03. Overall, it seems as though the Vgg16 transfer outperforms the scratch model on the training loss metric for every epoch. 


\subsection{Top 1 Accuracy}

\section{Plot 2: Top 1 Accuracy on Test Set}

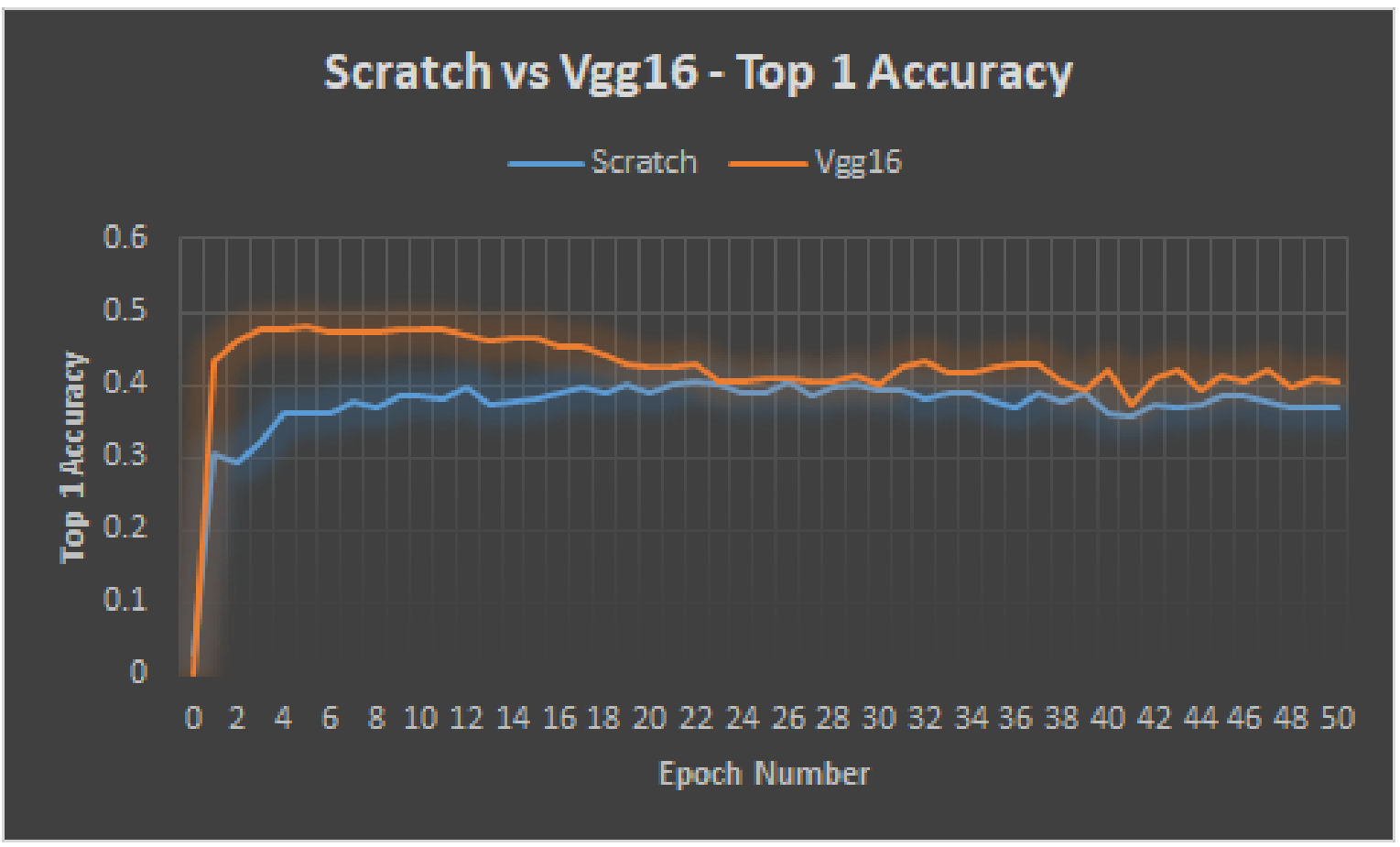

Table 2: Highlights of top 1 accuracy on test data.

\begin{tabular}{|l|l|l|l|l|l|}
\hline \multicolumn{7}{|c|}{ Top 1 Accuracy } \\
\hline Epoch \# & 0 & 1 & 5 & 22 & 50 \\
\hline Scratch & $2.95 \%$ & $30.4 \%$ & $36.2 \%$ & $40.5 \%$ & $36.7 \%$ \\
\hline Vgg16 & $0.3 \%$ & $43.3 \%$ & $48.2 \%$ & $42.9 \%$ & $40.6 \%$ \\
\hline
\end{tabular}

Top 1 Accuracy refers to the model's ability to identify the image correctly by accounting only the model's first prediction, this prediction is the output category with the highest probability value. In the Keras implementation, the predictions are presented in a vector of probabilities. That means that the model's predictions are determined based on the category with 
the greatest value. Then the prediction is compared with the label to determine correctness. From there, accuracy is calculated over the full dataset for every epoch. In the results shown in Table 2, top 1 accuracy is calculated on the test set.

Starting with epoch zero, this is where the models makes their predictions before training, we see very low top 1 accuracies for both models. This is to be expected because, at random, the models have a $1 / 50$ chance or $2 \%$ possibility to correctly identify the label values. We see the Vgg16 transfer model with a $0.3 \%$ accuracy and the scratch model with a $2.95 \%$ accuracy. Now, after the first epoch with training, we see that the Vgg16 transfer model takes the lead on epoch 1 with an immediate accuracy jump of $43.3 \%$, while the scratch model jumps to an accuracy of $30.4 \%$. This jump in accuracy signifies the rather drastic improvement of prediction accuracy post training.

Then, notice how the Vgg16 transfer model seems to reach a maximum accuracy of $48.2 \%$ on only epoch 5 . This is opposed to the scratch model's accuracy of $36.2 \%$ on that same epoch. Something else we see in the data after Vgg16's fifth epoch is the model's accuracy begins to drop rather significantly over the remainder of the 50 epochs. We also take into account that the scratch model's accuracy continues to rise until its maximum, on epoch 22, of accuracy $40.5 \%$. The Vgg16 transfer model's accuracy at that epoch is $42.9 \%$. After this point is where we start to see the models become very close in accuracy and begin to taper off and oscillate.

Next, looking at the models' final accuracies on epoch 50, we see that the Vgg16 transfer model gets $40.6 \%$ and the scratch model gets $36.7 \%$ accuracy. Both models dropped from their maximum accuracies and resulted fairly close on the final accuracy rate. In fact, the Vgg16 model differs from the scratch model on the 50th epoch by $3.9 \%$ accuracy. Then, when looking 
at best case for both models, Vgg16 had a maximum of $48.2 \%$ and scratch had a maximum of $40.5 \%$. The difference between the two best accuracies of each model is $7.7 \%$.

Overall, despite the drop in accuracy of the Vgg16 transfer model, it still remains higher in accuracy over the entire 50 epochs. In terms of Top 1 Accuracy, the Vgg16 transfer model performs better than the scratch model. The scratch model came close to the transfer model at points, however, did not manage to surpass the Vgg16 transfer model. As expected, the Vgg16 transfer model outperforms the scratch model on Top 1 Accuracy.

\subsection{Top 5 Accuracy}

Plot 3: Top 5 Accuracy on Test Set

\section{Scratch vs Vgg16 - Top 5 Accuracy}
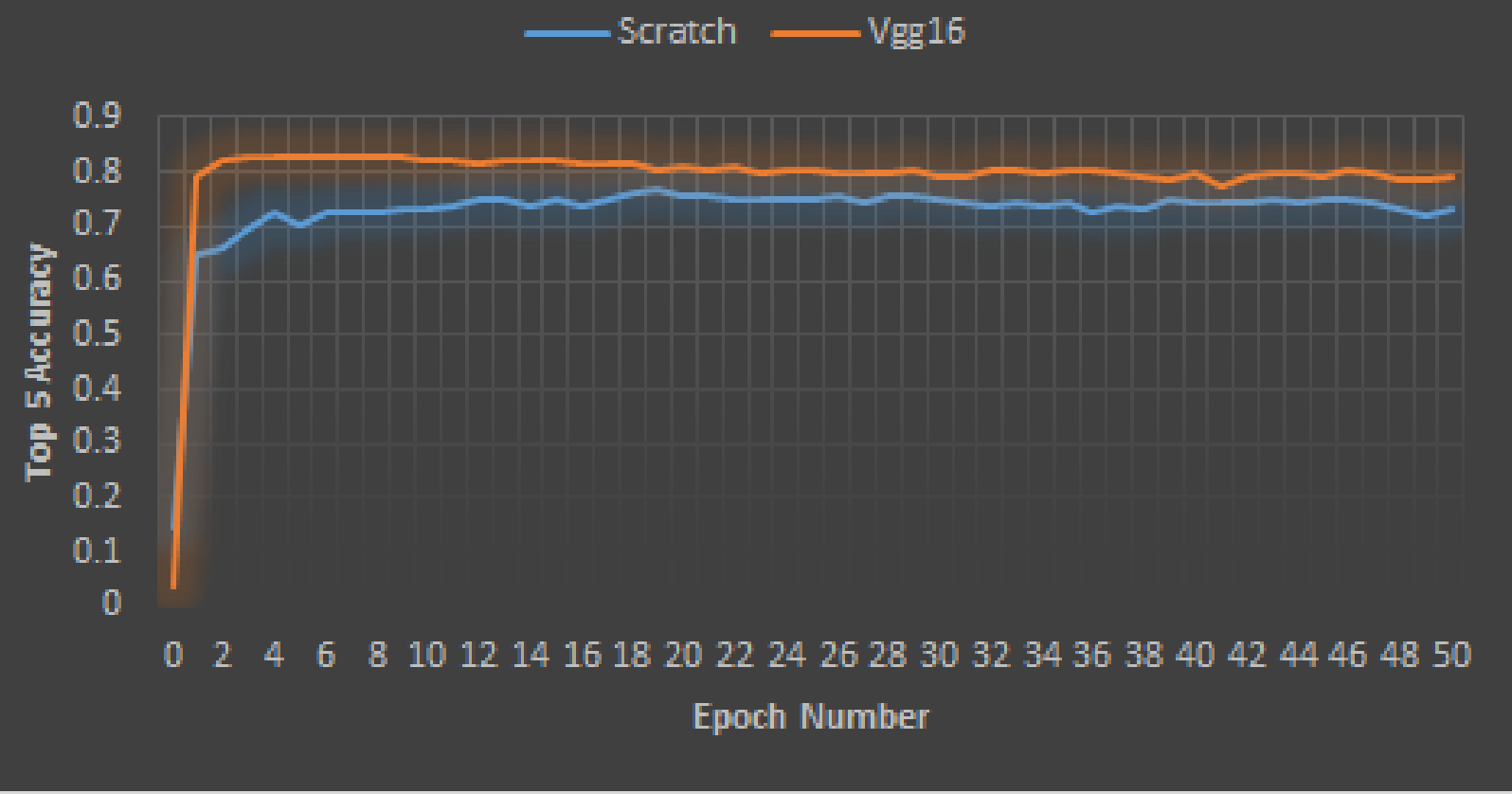
Table 3: Highlights of top 5 accuracy on test data.

\begin{tabular}{|l|l|l|l|l|l|}
\hline \multicolumn{7}{|c|}{ Top 5 Accuracy } \\
\hline Epoch \# & 0 & 1 & 5 & 19 & 50 \\
\hline Scratch & $14.4 \%$ & $64.65 \%$ & $70.3 \%$ & $76.6 \%$ & $73.0 \%$ \\
\hline Vgg16 & $3.7 \%$ & $79.45 \%$ & $83.0 \%$ & $80.45 \%$ & $79.45 \%$ \\
\hline
\end{tabular}

Top 5 Accuracy refers to the model's ability to identify the correct label of an image within its top 5 predictions. Similar to the Top 1 Accuracy, the Top 5 implementation is presented as a vector of probabilities. However, it selects five of its highest probabilities to compare with the label value, not just the highest one like top 1 accuracy. Also note, in the data shown in Table 3, Top 5 Accuracy is calculated on the test set.

Starting on epoch 0 , we see a top 5 accuracy of $3.7 \%$ from the Vgg 16 transfer model and $14.4 \%$ from the scratch model, this is the initial accuracy without any training. The expectation at random should give the model a 5/50 chance, so the expected no training accuracy is around $10 \%$. Then on epoch 1 , the first training, we see a tremendous improvement in model accuracies. The Vgg16 model jumps up to an accuracy of $79.45 \%$ and the scratch goes to a $64.65 \%$ accuracy. As they continue, both models continue to improve in accuracy.

Now, at epoch 5, we see that $\operatorname{Vgg} 16$ reaches its maximum of 83.0\%. At this same epoch, the scratch model is at $70.3 \%$ accuracy. From here, the Vgg16 transfer model starts to drop slightly and fluctuate for the remainder of the experiment. However, the scratch model continues rising until it reaches its maximum of $76.6 \%$ accuracy on epoch 19 . $\operatorname{Vgg} 16$ at this epoch had an accuracy of $80.45 \%$, a $2.55 \%$ drop from its own maximum. After this epoch, both models just continue to fluctuate until the end of the runs. 
By the 50th epoch, the scratch model concludes with a $73.0 \%$ accuracy and the transfer model with $79.45 \%$ accuracy. In terms of difference, the Vgg16 transfer has a $6.45 \%$ higher accuracy than the scratch model. On the models' best accuracies, Vgg16 transfer has $83.0 \%$ accuracy and scratch has $76.6 \%$ accuracy. The difference in their best case is $6.4 \%$.

Overall, the Vgg16 transfer model remains dominant in Top 5 Accuracy over the full 50 epochs of training. Not even once did the accuracies come close to overlap. Also, notice that the drop in accuracy rates of the models for this metric are less significant than those in the Top 1 Accuracy experiment. Once more, as expected, Vgg16 transfer outperforms the scratch model for the Top 5 Accuracy metric. 


\subsection{Model Predictions}

Figure 25: Top 5 Scratch Model Predictions
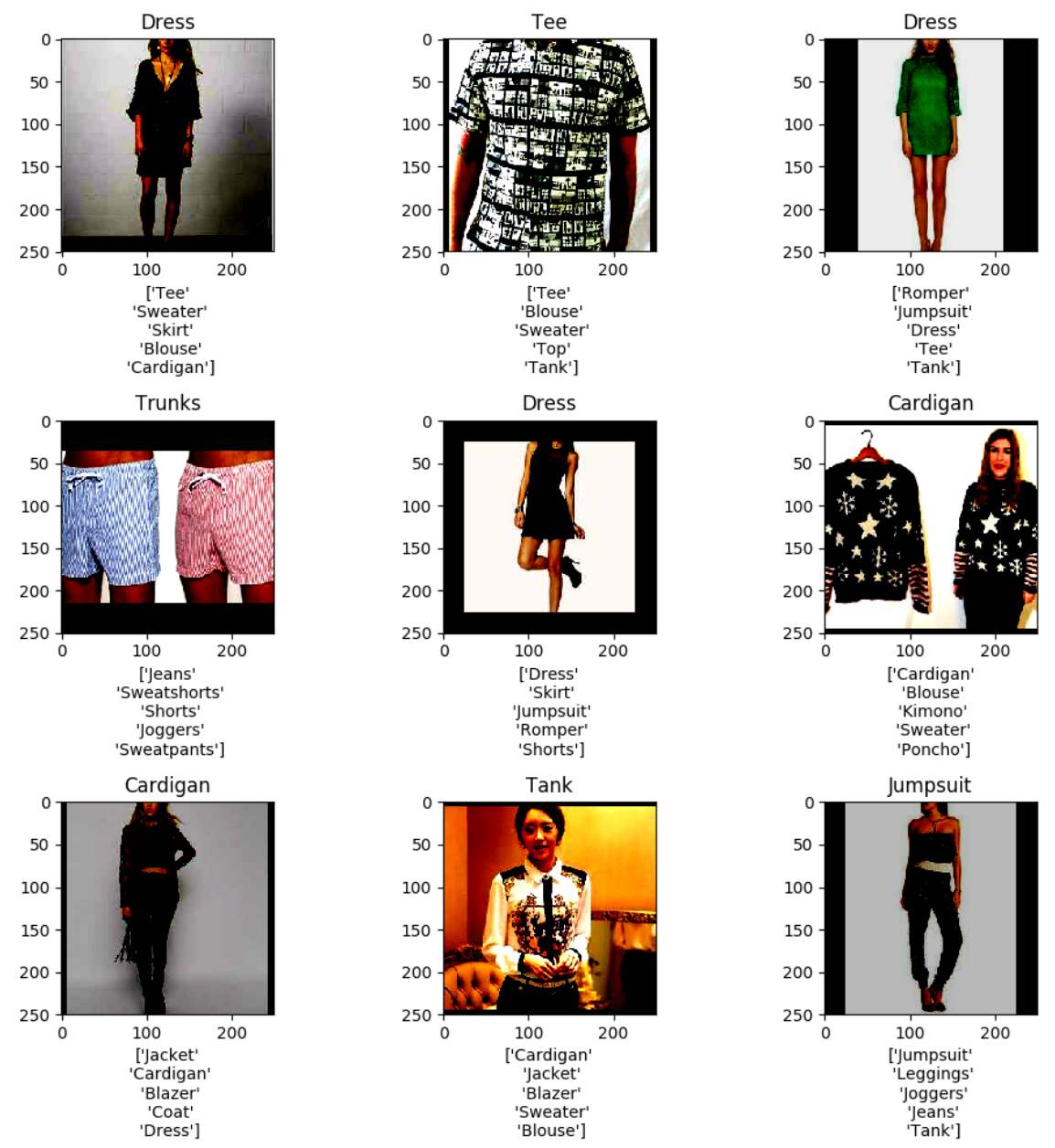

Figure 25 gives the scratch model's top 5 predictions on 9 randomly selected images from the testing set. Here, the images are cropped or padded to fit the height and width dimensions of $250 \times 250$. In the figure, we also see that the images have two sets of labels above and below each image. The label above the image is the "correct" category classification of the image. The 5 labels below the image in brackets are the model's top 5 predictions on the image. In terms of top 
1 accuracy, we see that the scratch model correctly identifies $4 / 9$ images correctly. In terms of top 5 accuracy, the scratch model correctly identifies $6 / 9$ images within its 5 predictions.

Figure 26: Top 5 Vgg16 Transfer Model Predictions
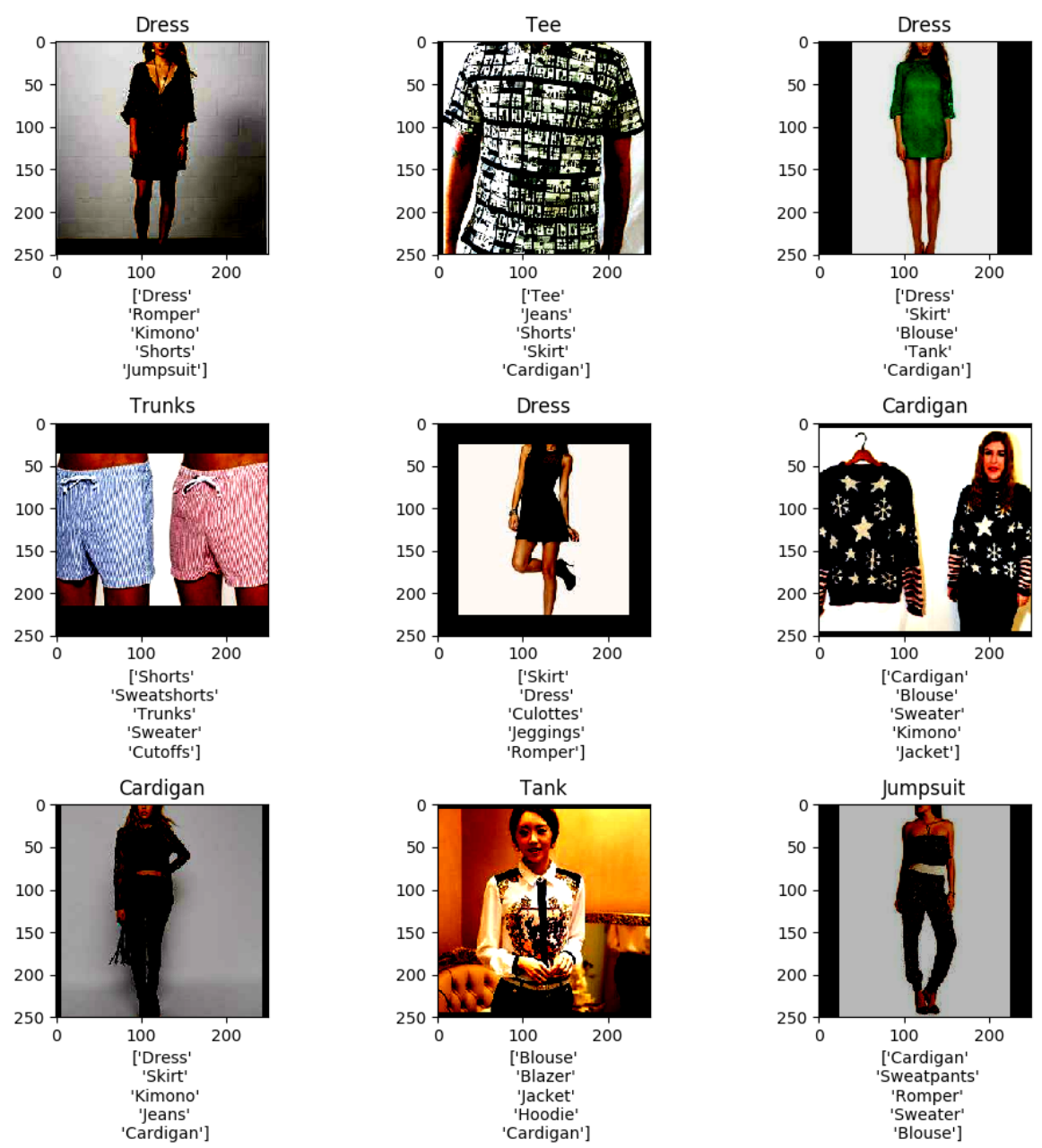

Figure 26 gives the Vgg 16 transfer model's top 5 predictions on the same 9 randomly selected images from the testing set. In terms of top 1 accuracy, we see that the Vgg 16 transfer model correctly identifies 4/9 images correctly. In terms of top 5 accuracy, the $\operatorname{Vgg} 16$ transfer model correctly identifies $7 / 9$ images within its 5 predictions. 


\subsection{Source(s) of Error}

Potential sources of error may include, but are not limited to the percentage of the data being only $5 \%$. This proportion of data may have been small enough that the Vgg16 transfer model could learn to memorize the labels or order of the data it was given. This means that the model would perform astoundingly on the training data, but fall flat when exposed to the test data. This is also referred to as overfitting to the training data, hence we see the the drop in model accuracy on the test data.

Now, the reason for such a small percentage is due to limitation on virtual memory. Another potential source of error could have been due to the modifications of hyperparameters on the scratch model. First, they could have been more fine-tuned before conducting the experiments. Secondly, the scratch model's architecture was modified from an initial 128 filters on the conv_2d_4 layer, from the Kratos project, to 80 filters because of the limitations on virtual memory.

\section{CONCLUSION}

From the results, we see that the Vgg16 transfer model outperforms the scratch model on each of the recorded metrics. The training loss at the start for the Vgg16 transfer model starts approximately $30 \%$ less than the scratch model and continues to grow in loss difference for approximately $3 / 5$ th of the training. We see that both models are decreasing in loss, but the Vgg16 transfer model drops at a faster rate and ends at a lower value. For accuracies, notice that on the first epoch of training, Vgg16's transfer model starts out with a top 1 accuracy rate of 
more than $10 \%$ over the scratch model. In terms of top 5 accuracy, the Vgg 16 transfer model also starts out with approximately $10 \%$ over the scratch model.

Now, also looking back at the top 5 predictions for both models, figures 25 and 26, we see that the scratch and $\operatorname{Vgg} 16$ transfer model get the same top 1 accuracy rate of $4 / 9=44.44 \%$. Comparing this to the Top 1 Accuracy plot, plot 2, we see that the ending accuracies for both these models are approximately $40 \%$. In terms of the Top 5 Accuracy, we see that the scratch model correctly identified 6/9 images, giving it approximately 66.66\% accuracy. On Vgg16 transfer model, it identified 7/9 images correctly, giving it approximately a $77.77 \%$ accuracy. If we look back at the plot comparing the models' top 5 accuracies, plot 3, we see that the $\operatorname{Vgg} 16$ transfer model ends with approximately a 10\% improvement over the scratch model for Top 5 Accuracy.

Overall, regarding top 5 predictions, the models do a fairly decent job identifying the images. For example, the image classified as "trunks", the models predicted "shorts" or "sweatshorts" within their top 5's. Honestly, I would have classified those "trunks" as "shorts" myself, so we see that the model's predictions aren't completely off even with a top 1 accuracy around $40 \%$.

So finally, after evaluating all the results of this experiment, we can confirm that the Vgg16 transfer model improves accuracy and minimizes the loss across individual epochs. Transfer learning does improve on the rate of accuracy improvement and speed of training per epoch iteration. According to the collected data, it seems as though the transfer model does its best within the first few epochs. Eventually, the scratch model does come close to meeting with the transfer learning's top 1 accuracy. However, the Vgg16 transfer model's accuracy tends to 
remain higher, even after it dropped from its initial high points. From the results, I conclude that models trained using transfer learning reach higher accuracies in fewer epochs than general models developed from scratch.

\section{FUTURE WORK}

Potential future work may include but is not limited to:

- Merging the models' predictions together by taking a weighted average of the results from both models, then calculating predictions to see how top 1 and top 5 accuracy are affected.

- Further developing the scratch model architectures to improve on individual model top 1 and top 5 accuracies. Specifically, I want to fine tune the scratch model to the point where it can outperform the Vgg16 transfer model on the Deep Fashion dataset. Then, once the scratch model outperforms the Vgg16 transfer model, I plan to train both model architectures on new datasets and compare their performances. Specifically, I want to know if the accuracies from training on the Deep Fashion dataset will persist when training on different datasets.

\section{KEYWORDS}

Artificial Intelligence, Machine Learning, Transfer Learning, Neural Networks (NNs), Convolutional Neural Networks (CNNs), Image Recognition, Computer Vision, Clothing Classification. 


\section{REFERENCES}

${ }^{[1]}$ Arel, I., Rose, D., \& Karnowski, T. (2010). Deep Machine Learning - A New Frontier in Artificial Intelligence Research [Research Frontier]. IEEE Computational Intelligence Magazine, 5(4), 13-18. doi: 10.1109/mci.2010.938364

${ }^{[2]}$ de Saint Laurent, C. (2018). In defence of machine learning: Debunking the myths of artificial intelligence. Europe 'S Journal Of Psychology, 14(4), 734-747. Doi:10.5964 /ejop.v14i4.1823

${ }^{[3]}$ Li, H., Parikh, N., \& He, L. (2018). A Novel Transfer Learning Approach to Enhance Deep Neural Network Classification of Brain Functional Connectomes. Frontiers In Neuroscience, 12, 1-12. doi: 10.3389/fnins.2018.00491

${ }^{[4]}$ Šajn, L., \& Kukar, M. (2011). Image processing and machine learning for fully automated probabilistic evaluation of medical images. Computer Methods And Programs In Biomedicine, 104(3), e75-e86. doi: 10.1016/j.cmpb.2010.06.021

${ }^{[5]}$ Lin, S., Kung, S., \& Lin, L. (1997). Face recognition/detection by probabilistic decision-based neural network. IEEE Transactions On Neural Networks, 8(1), 114-132. doi: $10.1109 / 72.554196$

${ }^{[6]}$ Stilgoe, J. (2017). Machine learning, social learning and the governance of self-driving cars. Social Studies Of Science, 48(1), 25-56. doi: 10.1177/0306312717741687

${ }^{[7]}$ Kowalski, A., Le, J., Emory, R., Lambert, S., Han, Y., Li, Y., \& Salah, Z. (2019). Kratos. Portland, OR. Retrieved from https://github.com/kratos-ai/Kratos

${ }^{[8]}$ Liu, Z., Luo, P., Qiu, S., Wang, X., \& Tang, X. (2019). DeepFashion: Powering Robust Clothes Recognition and Retrieval with Rich Annotations. Retrieved from 
https://www.cv-foundation.org/openaccess/content_cvpr_2016/papers/Liu_DeepFashion _Powering_Robust_CVPR_2016_paper.pdf

${ }^{[9]}$ van Gerven, M., \& Bohte, S. (2017). Editorial: Artificial Neural Networks as Models of Neural Information Processing. Frontiers In Computational Neuroscience, 11. doi:

10.3389 /fncom.2017.00114

${ }^{[10]}$ Torrey, L., \& Shavlik, G.K. (2009). Chapter 11 Transfer Learning.

${ }^{[11]}$ Das, S. (2017). CNN Architectures: LeNet, AlexNet, VGG, GoogLeNet, ResNet and more .... Retrieved from https://medium.com/@sidereal/cnns-architectures-lenet-alexnet-vgg -googlenet-resnet-and -more-666091488df5

${ }^{[12]}$ Tsang, S. (2018). Review: VGGNet — 1st Runner-Up (Image Classification), Winner (Localization) in ILSVRC 2014. Retrieved from https://medium.com/coinmonks/paper -review-of-vggnet-1st-runner-up-of-ilsvlc-2014-image-classification-d02355543a11

${ }^{[13]}$ Gershgorn, D. (2017). The data that transformed AI research—and possibly the world. Retrieved from https://qz.com/1034972/the-data-that-changed-the-direction-of-ai -research-and-possibly-the-world/

${ }^{[14]}$ Simonyan, K. \& Zisserman, A. (2014). Very Deep Convolutional Networks for Large-Scale Image Recognition. CoRR, abs/1409.1556.

${ }^{[15]}$ Descending into ML: Training and Loss $\mid$ Machine Learning Crash Course | Google Developers. (n.d.). Retrieved March 22, 2019, from https://developers.google.com/machine-learning/crash-course/ descending-into-ml/training-and-loss 
${ }^{[16]}$ Hao, Z. (2017, June 06). Loss Functions in Neural Networks. Retrieved from https://isaacchanghau.github.io/post/loss_functions/

${ }^{[17]}$ Agrawal, A. (2017, September 29). Loss Functions and Optimization Algorithms. Demystified. Retrieved from https://medium.com/data-science-group-iitr/ loss-functions-and-optimization-algorithms-demystified-bb92daff331c

${ }^{[18]}$ Rohrer, B. (n.d.). How convolutional neural networks (CNNs) work. Retrieved from https:/end-to-end-machine-learning.teachable.com/courses/how-deepneural-networks-work/lectures/9533964

${ }^{\text {[19] }}$ Parmar, R. (2018, September 02). Common Loss functions in machine learning. Retrieved from https://towardsdatascience.com/common-loss-functions-in -machine-learning-46af0ffc4d23

${ }^{[20]}$ Hassan, M. U. (2018, November 21). VGG16 - Convolutional Network for Classification and Detection. Retrieved from https://neurohive.io/en/popular-networks/vgg16/

${ }^{[21]}$ TensorFlow. (n.d.). Retrieved from http://www.tensorflow.org/ 